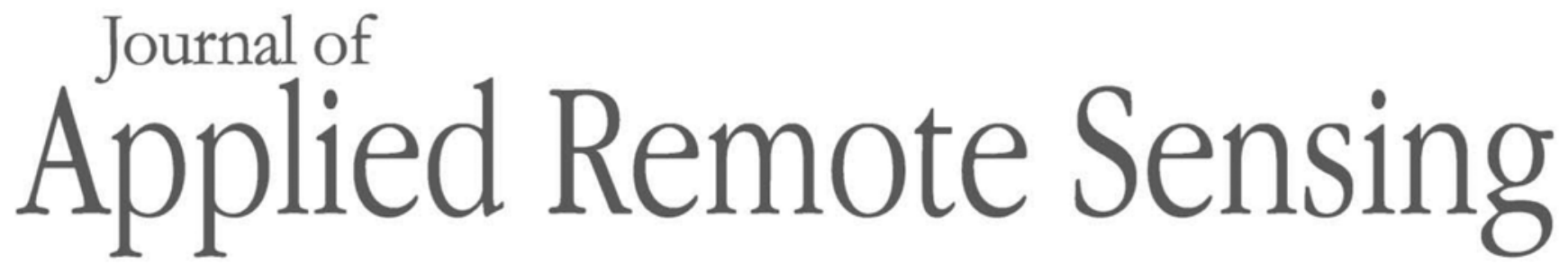

RemoteSensing.SPIEDigitalLibrary.org

\title{
Analyses of satellite ocean color retrievals show advantage of neural network approaches and algorithms that avoid deep blue bands
}

\author{
Ahmed El-Habashi \\ Sam Ahmed \\ Michael Ondrusek \\ Vincent Lovko
}




\title{
Analyses of satellite ocean color retrievals show advantage of neural network approaches and algorithms that avoid deep blue bands
}

\author{
Ahmed El-Habashi, ${ }^{\text {a,b }}$ Sam Ahmed, ${ }^{\mathrm{b}, *}$ Michael Ondrusek, ${ }^{\mathrm{c}}$ and \\ Vincent Lovko ${ }^{d}$ \\ ${ }^{a}$ Naval Research Laboratory, Remote Sensing Division, Washington, DC, United States \\ ${ }^{b}$ NOAA CREST Optical Remote Sensing Laboratory, City University of New York, \\ City College of New York, New York, United States \\ ${ }^{\mathrm{c}}$ NOAA/NESDIS 5830 University Research Court, College Park, Maryland, United States \\ ${ }^{\mathrm{d}}$ Mote Marine Laboratories, Sarasota, Florida, United States
}

\begin{abstract}
We have previously shown the advantage of using neural network (NN) inversion algorithms over other ocean color (OC) algorithms in Visible Infrared Imaging Radiometer Suite satellite retrievals of Karenia brevis $(K B)$ in the west Florida shelf (WFS). We now extend $\mathrm{NN}$ retrievals well beyond the WFS, to include both complex coastal and open ocean waters along the Florida and Atlantic coasts with a large dynamic range of chlorophyll- $a$ values. Most importantly, we add in situ radiometric measurements (which in contrast to satellite retrievals, are invulnerable to atmospheric transmission correction errors) as inputs to retrieval algorithms, permitting algorithm comparisons for in situ and simultaneous colocated satellite retrievals against sample measurements. Results unequivocally demonstrate the intrinsic efficacy and unfettered applicability of NN algorithms in widely varying waters beyond the WFS. Furthermore, they show that avoiding deep blue bands in retrieval algorithms significantly improves accuracies. Likely, rationales are that longer wavelengths (used with $\mathrm{NN}$ ) are less vulnerable to atmospheric transmission correction errors and to spectral interference by colored dissolved organic matter and nonalgal particles in more complex waters than deeper blue wavelengths (used with other algorithms), thereby arguing for development of OC algorithms using longer wavelengths. Finally, quantitative analysis of temporal, intrapixel, and sample depth variabilities highlights their important impact on retrieval accuracies. (C) The Authors. Published by SPIE under a Creative Commons Attribution 4.0 Unported License. Distribution or reproduction of this work in whole or in part requires full attribution of the original publication, including its DOI. [DOI: 10.1117/1.JRS.13.024509]
\end{abstract}

Keywords: ocean color; algorithms development; atmospheric correction; remote sensing reflectance; chlorophyll- $a$ retrievals; inherent optical properties; normalized fluorescence height; neural networks; Karenia brevis; harmful algal blooms; west Florida shelf.

Paper 180942 received Nov. 26, 2018; accepted for publication Mar. 29, 2019; published online May 2, 2019.

\section{Introduction}

In our previous work, ${ }^{1-4}$ results showed the efficacy of a neural network (NN) approach for detecting Karenia brevis $(K B)$ harmful algal blooms (HABs) in the west Florida shelf (WFS). The essence of the approach is the application of a standard multiband NN inversion algorithm, evolved and reported by us. ${ }^{1,5-8}$ This takes Visible Infrared Imaging Radiometer Suite (VIIRS) remote sensing reflectance $\left(R_{r s}\right)$ measurements at the 486, 551, and $671 \mathrm{~nm}$ bands [or 488, 555, and $667 \mathrm{~nm}$ bands for Moderate Resolution Imaging Spectroradiometer Aqua (MODIS-A)] as inputs. It then produces related inherent optical properties (IOPs) at $443 \mathrm{~nm}$ as its outputs, including the absorption coefficients of phytoplankton $\left(a_{p h 443}\right)$. The NN is used to generate an $a_{p h 443}$ image of the WFS, which is then converted into an equivalent chlorophyll- $a$ concentration $[\mathrm{Chl} a]$ image, using empirical relationships for specific chlorophyll- $a$ absorption values in the WFS, which have been determined from in situ measurements. ${ }^{9}$ Then, to obtain $K B$

*Address all correspondence to Sam Ahmed, E-mail: ahmed@ccny.cuny.edu 
HABs values from the VIIRS NN retrieved $a_{p h 443}$ image, two filter processes are applied. These are based on constraints known to be associated with $K B$ HABs in the WFS. These are: (i) low backscatter at $551 \mathrm{~nm}$ and (ii) a minimum permissible [Chl $a$ ]min threshold value ${ }^{10,11}$ and hence an equivalent minimum permissible value of $a_{p h 443} \leq a_{p h 443 \text { min }}$. Following the application of these two filter processes, the residual image shows $a_{p h 443}$ values that are compatible with both the above criteria for $K B \mathrm{HABs}$ and are convertible to $K B \mathrm{HAB}$ concentrations. In previously published work, ${ }^{1,2}$ VIIRS retrievals of $K B$ HABs in the WFS, using the NN technique, were compared with those obtained using other retrieval algorithms to evaluate its efficacy. All retrievals were evaluated, with the National Oceanic and Atmospheric Administration (NOAA) participation, against near coincident in situ cell count measurements available from the start of the VIIRS mission in January 2012 to 2016 using NASA/NOAA data. The results highlighted the important impact of short-term (15 to $20 \mathrm{~min}$ ) temporal variations on retrieval accuracies. ${ }^{1,2,4}$ These previous empirical comparisons also unequivocally showed that the NN technique exhibited the highest accuracy statistics for retrievals of $K B$ HABs in the WFS, closely followed by ocean color indexes $(\mathrm{OCI} / \mathrm{OCx})$, then more distantly by the red green chlorophyll index (RGCI), the generalized inherent optical property (GIOP), and the quasianalytical algorithm (QAA), particularly in optically complex and shallow waters. However, it should be noted that this previous work was restricted to the WFS. In this paper, we examine and more generally compare and extend the applicability of the NN technique to waters well beyond the WFS with larger dynamic ranges of chlorophyll- $a$ values and with much larger numbers of data points. In addition to the results of this extended comparison, significant results are also presented in two other areas. Highlights for all three areas are summarized below.

i. We extend evaluations and comparisons of NN retrievals of $a_{p h 443}$ and [Chl $\left.a\right]$ with retrieval from other OC algorithms to waters well beyond the WFS, including both complex coastal and open ocean waters, along the Florida and Atlantic coasts, with a large dynamic range of chlorophyll- $a$ values. Further and most importantly, in addition to retrievals from satellite measurements, we now also add coincident in situ radiometric measurements to obtain $R_{r s}$ inputs to retrieval algorithms. These in situ radiometric $R_{r s}$ measurements, in contrast to satellite $R_{r s}$ retrievals, are invulnerable to atmospheric transmission correction errors. This permits comparison of in situ radiometric measurement-based retrievals with simultaneous colocated satellite retrievals, with validation of both against coincident in situ sample measurements. This allows us to isolate different factors affecting algorithms retrieval accuracies and evaluate the intrinsic merits of different algorithms unencumbered by possibly inadequate or erroneous atmospheric transmission assumptions and/or satellite instrumental calibration limitations. The data for these extended studies, which are now freely available to the public and the scientific community, were obtained from NOAA dedicated VIIRS OC calibration and validation cruises, which we participated in. NN algorithm retrieval comparisons were carried out again with NOAA participation. While still empirical, much larger numbers of data points are used. The results unequivocally extend and demonstrate the efficacy of NN algorithms to widely varying waters beyond the WFS. The $\mathrm{NN}$ algorithm again exhibited higher retrieval accuracy statistics in comparisons with other algorithms. It was again closely followed by OCI/OCx, then more distantly by other algorithms, particularly in optically complex and shallow waters.

ii. Since it is conjectured that in satellite retrievals, it is the deep blue wavelengths that are more detrimentally affected by atmospheric correction inadequacies, the work reported here also zeroed in on impacts on algorithm retrieval accuracy when deep blue wavelengths are used for retrieving $R_{r s}$ values. For this work, we compared algorithm retrievals from the two best performing algorithms, NN and OCI/OCx against in situ sample measurements. These comparisons used in turn in situ radiometric $R_{r s}$ measurements and satellite $R_{r s}$ retrievals as inputs, with blue wavelengths, first at $443 \mathrm{~nm}$ (deep blue) and then at $486 \mathrm{~nm}$ (nondeep blue) in each of the algorithms. Comparisons of these two types of retrievals using 443 or $486 \mathrm{~nm}$ permit us to examine relative impacts at each wavelength of atmospheric correction inadequacies, as well as identify possible underwater spectral interference in colored dissolved organic matter (CDOM) rich and complex waters and generally evaluate the relative impacts for the deep blue and nondeep blue bands. The 
results unambiguously show that both satellite retrieval accuracies, as well as intrinsic retrieval accuracies from in situ radiometric measurements, are improved when deep blue wavelength measurements $(443 \mathrm{~nm})$ are avoided in favor of nondeep blue wavelengths $(486 \mathrm{~nm})$, thereby arguing for development and use of OC algorithms using the longer wavelengths.

iii. Finally, quantitative analysis of temporal, intrapixel, and sample depth variabilities highlights their important impact on retrieval accuracies.

\section{Background}

\subsection{NN Algorithm}

For the development of the NN algorithm, ${ }^{1,2,4,12-15}$ a synthetic dataset of 20,000 IOPs was randomly simulated within the very wide range of parameters exhibited in the NASA Bio-optical Marine Algorithm Dataset. ${ }^{12}$ These randomly generated synthetic IOPs ${ }^{13-17}$ were then used as inputs to a four component bio-optical model, ${ }^{7,16,17}$ which in conjunction with a HydroLight based ${ }^{18}$ parameterized forward model, described in Lee et al., ${ }^{16}$ and produced 20,000 sets of $R_{r s}$ values at 486, 551, and $671 \mathrm{~nm}$ (for VIIRS) and at 488, 555, and $667 \mathrm{~nm}$ for MODIS. The NN was trained on 10,000 of these values and tested on the 10,000 remaining subset, to solve the inverse problem ${ }^{19}$ of retrieving physical variables, including $a_{p h 443}$, from $R_{r s}$ values at 486,551 , and $671 \mathrm{~nm}$, and at 488,555 , and $667 \mathrm{~nm}$. The algorithm is a standard multiband NN inversion algorithm that takes $R_{r s}$ input at 486, 551, and $671 \mathrm{~nm}$ wavelengths, which are not greatly impacted by atmospheric corrections. Output of IOPs, namely absorption coefficients of phytoplankton $\left(a_{p h}\right)$, dissolved organic matter $\left(a_{g}\right)$, and nonalgal particulates $\left(a_{d m}\right)$ as well as the particulate backscatter coefficient $\left(b b_{p}\right)$, all at $443 \mathrm{~nm}$, which is at the peak of $a_{p h}$ and thus exhibits most variation. Detailed descriptions of the NN are given in Refs. 1 and 4-8. A brief description of $\mathrm{NN}$ as well as the necessary parameters for its implementation are given in Secs. 7.1 and 7.2.

\subsection{Summary of Previous Results-NN VIIRS HABs Retrieval Match-Ups in the WFS against Near Simultaneous In Situ Cell Count Measurements Compared with Retrievals Using Other Algorithms}

To place the results presented in this paper in a proper context, a brief summary of previous results (1) and (2) is presented here. In that work, which was restricted to the WFS, retrievals of $K B$ HABs from VIIRS satellite observations were matched with simultaneous or near simultaneous in situ sample measurements obtained over a 4 year period, 2012-2016, for which there were available VIIRS observations and near concurrent in situ data, collected by the Florida Fish and Wildlife Conservation Commission (FWC). For these observations, the accuracy of retrievals using the NN algorithm was compared to the accuracy of retrievals obtained using three other available $\mathrm{OC}$ retrievals algorithms: (i) the $\mathrm{OCIOOCx^{20,21 }}$ are the default NASA products ${ }^{22}$ that are used to retrieve the near-surface chlorophyll- $a$ concentrations [Chl $a$ ]; the product uses a combination of the OCX and OCI algorithms; (ii) the GIOP model, ${ }^{23,24}$ the model estimates unified IOPs from different semianalytical algorithms; (iii) the QAA, ${ }^{16,25,26}$ developed by Lee et al. ${ }^{16}$ to derive the absorption and backscattering coefficients by analytically inverting the spectral remote-sensing reflectance $\left[R_{r s}(\lambda)\right]$; and (iv) the RGCI is an regional algorithm ${ }^{27}$ that retrieves $[\mathrm{Chl} a]$ from observations of MODIS-A and VIIRS bands.

The results of the comparisons supported the conclusion that at least for these preliminary and somewhat limited datasets, the NN satellite retrievals exhibit significantly better performances against the in situ measurements, for both the longer (100 min) and, more importantly, the shorter (15-min) overlap time windows. This was observed both in terms of higher coefficient of determination $\left(R^{2}\right)$ and in terms of lower errors against the in situ measurements. It is also seen that reducing the time window from 100 to 15 min between satellite and in situ observations very significantly increased the accuracy between VIIRS retrieved [Chl $a$ ] and in situ measured $K B$ cell counts. We believe that this reflects temporal changes in the HABs scene being observed. These changes are clearly greater over the 100-min time window than for the 15-min window. 
These changes, can be quite rapid, ${ }^{28}$ were confirmed by our field measurements of $K B$ HABs in the WFS and reported in Ref. 2, where it was found that changes of the order of $30 \%$ in HABS cell concentrations could typically occur (up or down) in tens of minutes whether due to drift or up- or downwelling effects. ${ }^{2}$ These temporal effects in the WFS are now explored quantitatively in Sec. 4.4 below.

\section{Methods}

This section describes the approach and methodology followed in this paper to arrive at results in three areas:

\subsection{Examination of General Applicability and Advantages of NN Approach in Different Waters Types Beyond the WFS}

To answer questions previously raised regarding general applicability and potential advantages of NN approach in waters beyond the WFS, in this paper, we extend NN retrieval comparisons to locations well beyond the WFS, to include both complex coastal and open ocean waters along the Florida and Atlantic coasts, which encompass different water types with a large dynamic range of chlorophyll- $a$ values. The NN retrievals are now compared to retrievals using the following algorithms: (i) the OCI $\backslash \mathrm{OCx},{ }^{20,21}$ (ii) the QAA, ${ }^{16,25,26}$ and (iii) the RGCI. ${ }^{27}$ These measurements and retrievals were carried out in NOAA cruises and our own cruises with Mote Marine Laboratory in the WFS over the 2014 to 2018 period. Most importantly, in these comparisons, in addition to extending locations and water types studied, we now also added in situ $R_{r s}$ radiometric measurements, to complement the in situ sampling. Since these $R_{r s}$ measurements are in situ, they are not subject to the impact of possibly inadequate (or incorrect) correction procedures that are applied to atmospheric transmission assumptions and that can erroneously impact satellite $R_{r s}$ retrievals and hence retrieval accuracies. Instead, by using these in situ $R_{r s}$ measurements as inputs to the different retrieval algorithms, the retrieval comparisons obtained in this manner indicate the intrinsic retrieval accuracies and efficacy of the algorithms being compared, without the impact of possibly inadequate and erroneous atmospheric transmission assumptions.

In addition to the above retrieval comparisons obtained from in situ radiometric measurements, comparisons were also made of available simultaneous satellite retrievals using the different algorithms of the same locations. Besides permitting us to rank the satellite retrieval accuracies and efficacies of NN and other algorithms for waters beyond the WFS, this permits us to examine and isolate the impacts of inadequate or incorrect assumptions for an atmospheric transmission on these satellite retrieval accuracies.

Figure 1 shows the location of all open ocean, coastal, bloom, and nonbloom waters, where in situ radiometric measurements and sample measurements were made, along with some coincident satellite measurements. A total of 83 coastal and open ocean measurements were obtained from the NOAA VIIRS calibration and validation cruises ${ }^{29-31}$ over the 2014 to 2016 period as well from in situ measurements that include KB blooms waters from our 2017 and 2018 WFS field campaigns with Mote. The comparisons also added examination and comparison of retrieval statistics for different retrieval algorithms as a function of distance from the coasts.

For comparisons of retrieval accuracies of NN, OCI/OCx, QAA, and RGCI algorithms, the orthogonal linear regression approach (OR) was used to determine the coefficient of determination, $R^{2}$, and errors $(\varepsilon)$. The error $(\varepsilon)$ is calculated as the sum of orthogonal distances and assumed to exist for both variables. OR estimates of $x$ on $y$ will minimize the orthogonal distance from the observed data points to the regression line: ${ }^{32}$

$$
\sum\left(y_{i}-\beta_{0}-\beta_{1} x_{i}\right)^{2}
$$

where $\beta_{0}$ and $\beta_{1}$ are intercept and slope. The OR estimate of the slope is as follows:

$$
\hat{\beta}_{1}=\frac{S_{Y Y}-S_{X X}+\sqrt{\left(S_{Y Y}-S_{X X}\right)^{2}+4 S_{X Y}^{2}}}{2 S_{X Y}},
$$



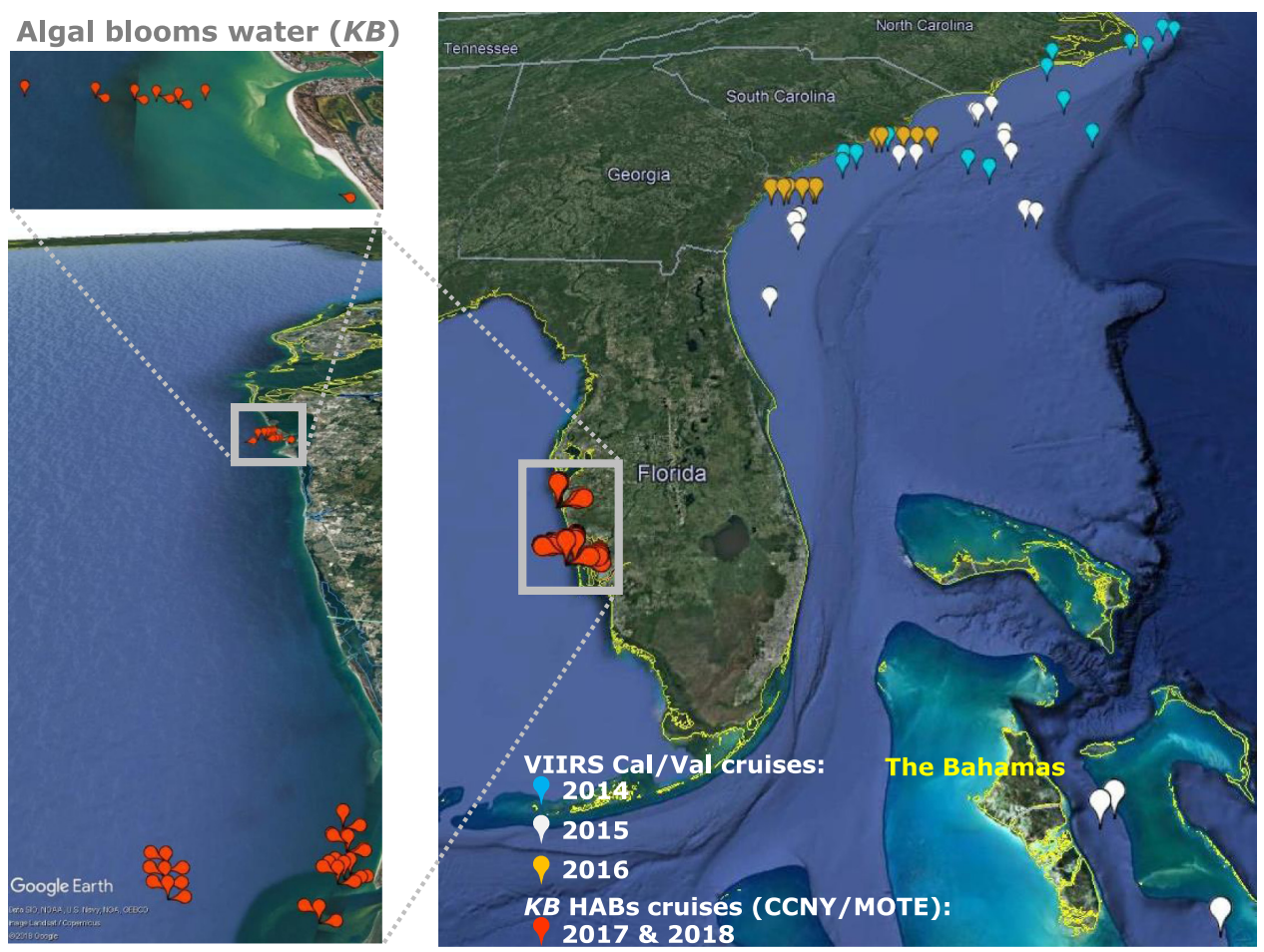

Fig. 1 Locations for in situ radiometric and sample measurements used in retrieval comparisons.

where, $S_{X X}$ and $S_{Y Y}$ are the covariances for $X$ and $Y$, respectively, and $S_{X Y}$ is the correlation for $X$ and $Y$.

The average magnitude errors were also calculated, using the mean absolute error (MAE), where all individual differences have equal weights. MAE calculates the average over the tested sample of the absolute differences between retrieved $\left(y_{j}\right)$ and actual measured $\left(\hat{y}_{j}\right)$ values. The MAE is defined as follows:

$$
\mathrm{MAE}=\frac{1}{n} \sum_{j=1}^{n}\left|y_{j}-\hat{y}_{j}\right|
$$

where $n$ is the number of observations.

\subsection{Impact of Deeper Blue Wavelengths on Retrieval Performance}

In the present work, satellite retrievals using different algorithms comparisons were carried out not only against coincident in situ sampling, but, as discussed above, also now extended to comparisons with retrievals using in situ surface and below surface coincident $R_{r s}$ radiometric measurements as inputs to the retrieval algorithms. Since it is conjectured that in satellite retrievals, it is the deep blue wavelengths that are more significantly affected by atmospheric correction inadequacies, we next zeroed in on the impact on algorithm retrieval accuracy when deep blue wavelengths are used for retrieving $R_{r s}$ values. For this work, we compared algorithm retrievals from by far the two best performing algorithms, NN and OCI/OCx against in situ sample measurements, using both in situ radiometric measurements and satellite $R_{r s}$ retrievals, as inputs, with each of the algorithms using as blue wavelengths either $443 \mathrm{~nm}$ (deep blue) or $486 \mathrm{~nm}$ (nondeep blue). These combined comparisons allow us to isolate the impact of atmospheric correction inadequacies, as well as identify possible underwater spectral interference in CDOM rich and complex waters. To carry out these comparisons, the NN algorithm, which normally uses $R_{r s}$ inputs at 486,551 , and $671 \mathrm{~nm}$, was retrained to use $R_{r s}$ at $443 \mathrm{~nm}$ instead of the $486 \mathrm{~nm}$ as input. Comparisons could then be carried out of both satellite and in situ radiometric retrievals using the same $\mathrm{NN}$ algorithm using the $R_{r s} 486 \mathrm{~nm}$ input or the deeper blue $R_{r s}$ input at $443 \mathrm{~nm}$ (to isolate relative impacts of inadequate atmospheric corrections, expected to be 
El-Habashi et al.: Analyses of satellite ocean color retrievals show advantage...

larger at $443 \mathrm{~nm}$ and underwater spectral interferences). These comparisons are also repeated for the OCx algorithm, which has options for either $443 \mathrm{~nm}$ or 486 inputs.

\subsection{Analysis of Temporal Changes, Sampling Depths and Intrapixel Variations of $K B$ HABs}

Our previous results, summarized in Sec. 2.2 above and reported in Refs. 1 and 2, implied that the accuracy and correlation of match-ups of VIIRS $K B$ HABs retrievals against near simultaneous sample measurements in the WFS were very vulnerable to relatively short-term temporal changes in the HABs scene being observed. We extend the quantitative examination of the impact on retrievals of these temporal variations, as well of variations with sampling depths and inter/intra-pixel variations by means of detailed in situ field sample and radiometric measurements in the WFS in conjunction with Mote Marine Laboratories in 2017 and 2018. Measurements were made along several transects, typically 2 to $3 \mathrm{~km}$ in length, off Sarasota, Florida. Many of the measurements were made at stations subpixel distances apart (generally $300 \mathrm{~m}$ ) on an outward leg and were then repeated for the same stations as closely as possible on a return leg. Radiometric and sample measurements were made at different depths: $0.1,0.5$, and $1 \mathrm{~m}$ for each station. Chlorophyll concentrations were obtained by high performance liquid chromatography (HPLC) analyses and $K B$ cell counts obtained by microscope analyses at Mote Marine Laboratories. We also examined images of $K B$ HABs retrievals from consecutive VIIRS and MODIS satellite overpasses of the WFS to support the conclusion of the observed short-term changes.

\section{Results}

The section is organized as follows: Sec. 4.1 represents evaluating and confirming the efficacy of NN OC retrievals beyond the WFS by extending comparisons with other OC algorithms to different water types in open ocean and complex coastal waters along the Florida and Atlantic coasts; Sec. 4.2 represents examining the impact of the use of deeper blue bands on satellite retrieval accuracies, and the resultant advantages of algorithms that avoid deep blue bands; Sec. 4.3 represents quantitative examination of temporal, sampling depth and intrapixel variabilities in $K B$ HAB waters of the WFS and their impact on retrieval accuracies; Sec. 4.4 represents satellite image retrievals and observations of variabilities in WFS.

\subsection{Evaluating and Confirming the Efficacy and Advantages of NN OC Retrievals Beyond the WFS by Extending Retrieval Comparisons with other OC Algorithms to Different Water Types in Open Ocean and Complex Coastal Waters along the Florida and Atlantic Coast}

In this section, NN OC retrievals are compared with OCI/OCx, QAA, and RGCI retrievals. The first set of comparisons uses in situ radiometric measurements as inputs to retrieval algorithms. The second set of comparisons, shown later below, compares the VIIRS satellite retrieval directly. The use of in situ radiometric inputs first eliminates concerns of possible atmospheric correction inadequacies associated with satellite retrievals. All comparisons are also extended to include a large variety of water beyond the WFS, including open ocean, complex coastal, and $K B$ bloom waters, covering a large dynamic range of chlorophyll- $a$ values. Field measurements were obtained from several field campaigns along the Florida and Atlantic coasts, as discussed in Sec. 3.1 above. Figure 1 shows the location of the 83 data points from these measurements.

Figure 2 shows retrievals and related statistics obtained with the different algorithms using in situ radiometric $R_{r s}$ measurements as inputs, plotted against sample measurements for all 83 stations, as shown in Fig. 1 above. This includes 40 complex coastal water measurements and 43 open ocean measurements, both from the WFS and Atlantic coasts.

Performance of the different algorithms was also examined by comparing the residuals (difference between retrieved values and sample measurements) at varying distances from the 


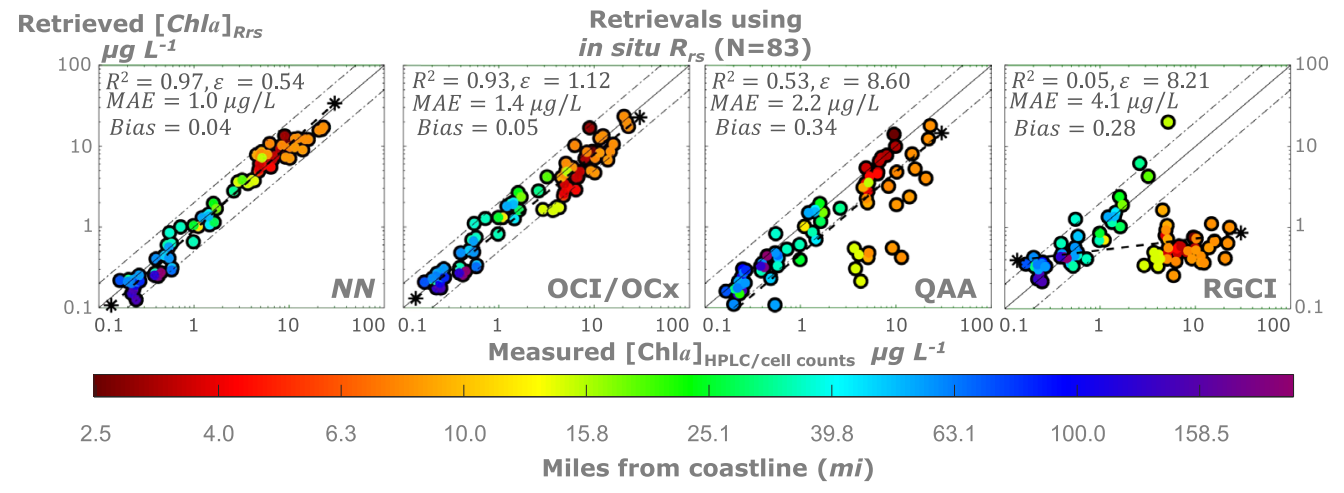

Fig. 2 Results for NN, OCl/OCx, RGCl, and QAA retrievals in the WFS and Atlantic coasts obtained using in situ radiometric measurements as inputs plotted against sample measurements for 83 stations, including 40 coastal and bloom water measurements, as well as 43 open ocean measurements. Color bar relates retrieved [Chla] statistics to varying distance from the shoreline.

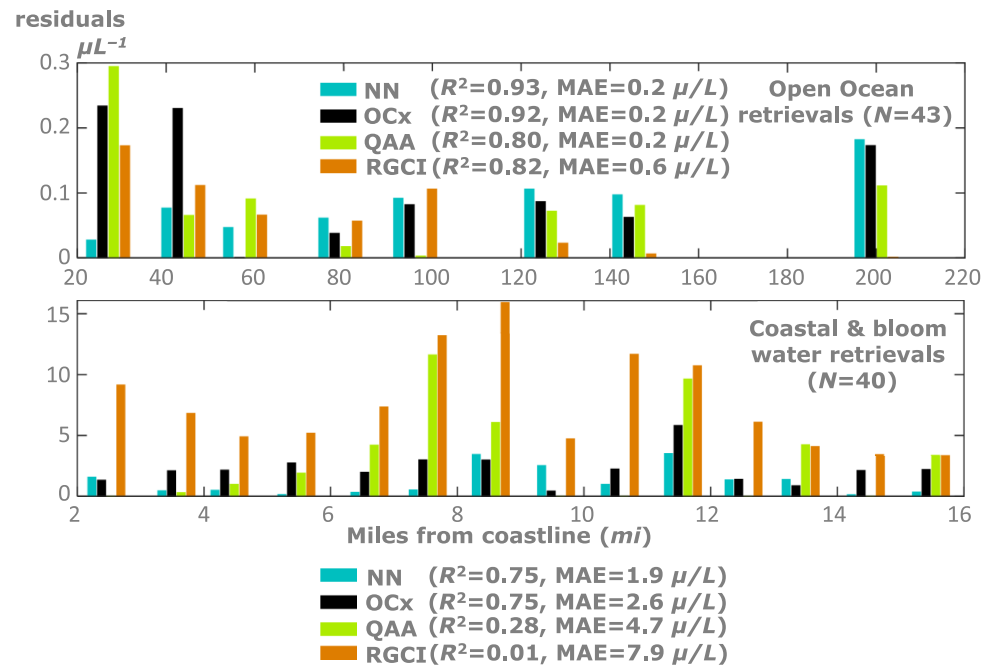

Fig. 3 Residuals (difference between retrieved values and sample measurements) at varying distances from the shoreline for the different algorithms.

shoreline. These are shown in Fig. 3 for both open ocean and complex coastal waters of the Atlantic coasts and $K B$ HABs water in WFS. In both cases, measured in situ radiometric $R_{r s}$ and HPLC samples values are used.

As can be seen from Figs. 2 and 3, for the coastal water regions located 2 to 16 miles from the shoreline, the $\mathrm{NN}$ algorithm generally exhibited the highest $R^{2}$ correlations, the lowest retrieval errors $(\varepsilon)$, and the lowest MAE of $1.9 \mu \mathrm{g} \mathrm{L}^{-1}$, closely followed by $\mathrm{OCx}$, then more distantly by QAA and RGCI, particularly in optically complex and shallow waters. For the open ocean regions, the order of retrieval accuracies was again $\mathrm{NN}$ followed by $\mathrm{OCx}$ and then QAA and RGCI. The regression lines in Fig. 2 seem to improve for $\mathrm{OCI} / \mathrm{OCx}$ for lower retrieved chlorophyll- $a$ values and in NN for the higher retrieved chlorophyll- $a$ values. The fact that for the more complex WFS waters with $K B \mathrm{HABs}$, the $\mathrm{NN}$ algorithm performs better, which would tend to support the notion that higher accuracies of $\mathrm{NN}$ retrievals may be at least partially due to the use of longer $R_{r s}$ input wavelengths for NN, than the deeper blue wavelengths used in other algorithms, thereby possibly lessening the impact on measured $R_{r s}$ values of spectral interference by dissolved organic matter CDOM, and nonalgal particles (both expected in more complex waters). It should also be noted that while the $\mathrm{NN}$ approach does not require measurements at the shorter wavelength channels, which are typically impacted the most by atmospheric correction difficulties and possible underwater spectral interference (CDOM/NAP) in coastal waters, the NN output of $\left(a_{p h}\right)$ is at the 443-nm band, which is at 
the peak of phytoplankton absorption, and thus exhibits the most sensitive variation in natural water. This contributes significantly to the sensitivity and accuracy of the NN approach to effectively retrieve the $a_{p h}$ (443) parameter, particularly in optically complex waters, without the atmospheric correction vulnerabilities, which would occur if actual satellite measurements in the deep blue (443 nm) band were required. In any case, it can be unambiguously concluded from these results that the intrinsic efficacy of $\mathrm{NN}$ retrieval algorithms extends well beyond the confines of the WFS to widely varying waters algorithms.

We next compare VIIRS satellite retrievals using different algorithms with colocated and concurrent in situ sample measurements. We therefore looked for match ups from the NOAA VIIRS Calibration and Validation cruises $^{29-31}$ on the Atlantic coasts, over the 2014 to 2016 period, as well as from our 2017 and 2018 field campaigns in the WFS. The conditions stipulated for match-up were that pixel centers were 0.3 miles or less from the in situ measurement location. This is an empirical approach to ensure that the pixel values could be reasonably assumed to reflect the related in situ measurements, more specifically for the case of bloom pixels, and hence reduce potential impact of patchiness ${ }^{33}$ within the pixel $\left(0.7\right.$ and $1.0 \mathrm{~km}^{2}$ correspond to pixel size for nadir observation with VIIRS and MODIS, respectively). Pixels were also excluded from the match-up comparisons if they had been flagged for any of the following: land, clouds, failure in atmospheric correction, stray light, bad navigation quality, both high and moderate glint, negative Rayleigh corrected radiance, viewing angles greater than $60 \mathrm{deg}$, and solar zenith larger than $70 \mathrm{deg}$, as well as any pixels, which had water leaving radiance spectra with negative values in any one wavelength. Chlorophyll- $a$ and $K B$ sample measurements had to be made at less than $1-\mathrm{m}$ depth. It should also be noted that [Chla] of $1 \mu \mathrm{g} \cdot \mathrm{L}^{-1}$ is equivalent to $\sim 10^{5} \mathrm{~KB}$ cells $\cdot \mathrm{L}^{-1}$. ${ }^{34}$

When comparisons were being made between retrievals using NN, OCI/OCx, QAA, and RGCI algorithms, it was found that there were 41 match-ups of available in situ measurements that satisfy the match-up conditions for the satellite observations stipulated above. Figure 4 above shows the wide variety of $R_{r s}$ values for all 41 match-ups obtained from VIIRS measurements and in situ radiometric measurements. These include measurements from complex coastal and open ocean waters in the WFS and Atlantic coasts. VIIRS satellite retrievals obtained using NN, OCI/OCX, QAA, and RGCI algorithms are shown in Fig. 5. The top row shows the available satellite match-ups retrievals compared against in situ sample measurements. As can be seen, NN and OCX satellite retrievals achieve much better accuracies against in situ sample measurements than the retrieval accuracies achieved by the QAA and RGCI algorithms, at least for this widely varied combination of waters. We would note that it is quite possible that in different specific water types, performance by the other algorithms may be improved beyond the results shown here for results from widely varying waters. For instance, in the results shown in Ref. 2, where tests were more restricted to $K B$ HABs retrievals in the WFS while NN still performed considerably better than other algorithms, it was again followed, in that case, less closely by OCI/OCx and then by RGCI, which in those waters achieved accuracies, still behind but closer to those of OCI/OCx and with accuracies better than those attained for the widely varying waters studied in this paper. In any case, the latest results presented here again unambiguously confirm

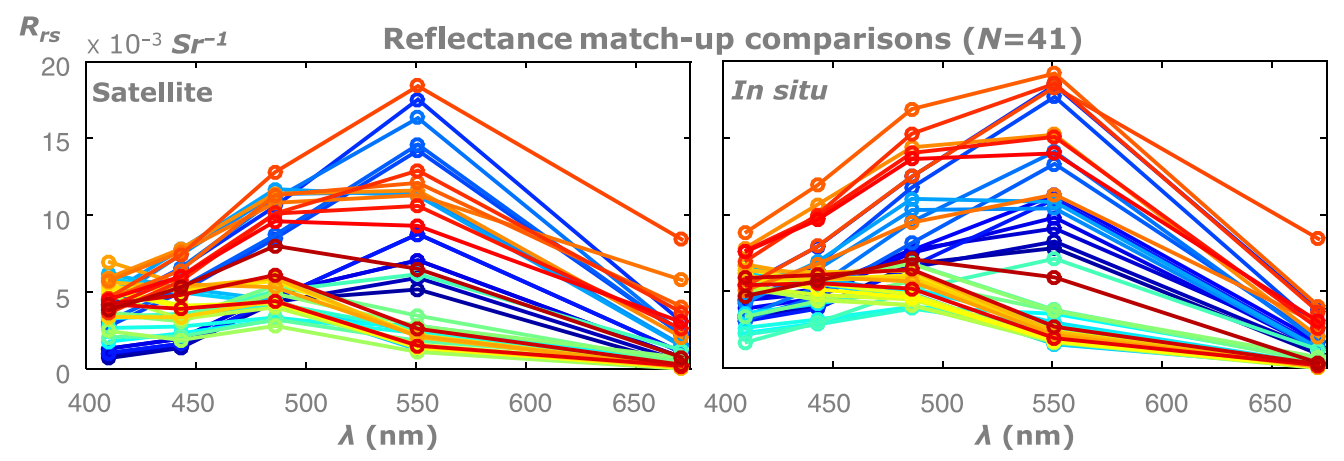

Fig. 4 Shows a total of $41 R_{r s}$ from both VIIRS satellite and in situ radiometric measurements covering a variety of water types over the WFS and the Atlantic coast sites. Color code is used to indicate the difference water types. Same color code is used for satellite and in situ figures. 


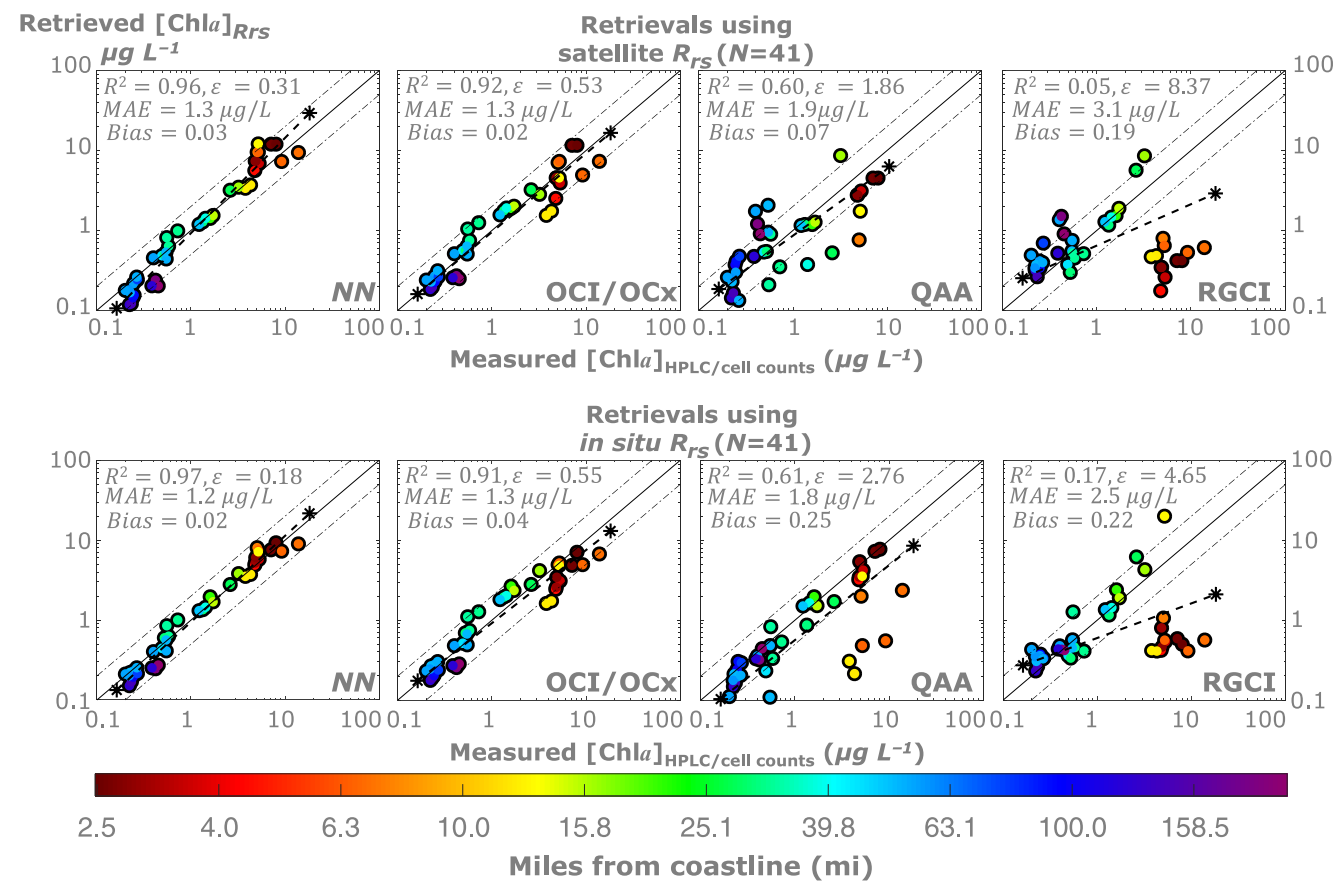

Fig. 5 Top row: satellite retrievals against in situ sample measurement. Bottom row: same algorithm retrievals, with same match-up locations, but using in situ radiometric measurements as inputs. Color bar relates retrieved [Chla] statistics to varying distance from the shoreline.

the efficacy of NN retrievals for varied and different water types beyond the WFS and argue for its adoption as a product for such retrievals.

We again compare retrievals for the same 41 locations but now use in situ radiometric measurements as inputs into the algorithms, bottom row, Fig. 5. These show very similar retrieval accuracies to those of the satellite retrievals, and that at least for these waters, inadequate atmospheric corrections did not significantly affect $\mathrm{NN}$ and OCx retrieved $R_{r s}$ values and retrieval accuracies (since both algorithms are using nondeep blue 486-nm inputs). Greater impact is observed ( $\varepsilon$ improved from 2.76 to 1.86 ) for the QAA retrievals, which use deeper blue band inputs at 410 and $443 \mathrm{~nm}$, with MAE showing some improvement, which is negatively constricted by the large number of outliers in the data for the in situ radiometric input retrievals. However, the improvements are more clearly seen in the reduced spread of the data points. In the case of RGCI, it is seen that retrieval accuracies are much lower than for the other algorithms, which is possibly due to the fact that RGCI is a regional specific algorithm intended for the WFS, and its behavior for the much larger variety of waters compared above is severely limited. Conclusions on the differences observed for in situ and satellite retrievals in the above figures are therefore difficult to assess in the case of RGCI. The relative impacts of deep blue $486 \mathrm{~nm}$ and nondeep blue $486 \mathrm{~nm}$ on retrieval accuracies are examined in more detail in Sec. 4.2, where retrievals with the same algorithms using deep blue and nondeep blue wavelengths are compared and unambiguous results obtained.

\subsection{Examining the Impact of the Use of Deeper Blue Bands on Satellite Retrieval Accuracies, and the Resultant Advantages of Algorithms that Avoid Deep Blue Bands}

The generally better performance of $\mathrm{NN}$ satellite retrievals against in situ sample measurements, particularly in HAB rich and complex coastal waters, is conjectured by us to be partially due to the relative invulnerability of the longer wavelengths used $(486,551,671 \mathrm{~nm})$ in $\mathrm{NN}$ to atmospheric correction inadequacies that impact shorter wavelengths used in other algorithms, particularly for closer in-shore waters. To more directly examine possible atmospheric correction inadequacy impacts, comparisons were made of retrievals using simultaneous in situ radiometric 
and satellite observations evaluated against in situ sample measurements-for different water regions-both in-shore and off-shore, and for algorithms using deep blue and nondeep blue wavelengths. These were carried out by examining retrievals accuracies obtained using NN and OCI/OCx algorithms applied to the previous set (Sec. 4.2 above) of 41 measurements, including $K B \mathrm{HABs}$, complex coastal and open ocean waters. In one set of measurements, $443 \mathrm{~nm}$ was used for the $R_{r s}$ input in the deep blue, and in the other set, $486 \mathrm{~nm}$ for the $R_{r s}$ input (for comparison as the not deep blue input). For the purpose of these comparisons, the NN algorithm was retrained and adapted to use $R_{r s}$ at $443 \mathrm{~nm}$ instead $486 \mathrm{~nm}$ as an input. OCx already has NASA provisions for using either 443 or $486 \mathrm{~nm}$ as input. These retrieval comparisons were carried out with one set using the in situ radiometric measurements as inputs (which are invulnerable to atmospheric correction inadequacies), to the retrieval algorithms, whereas the other set examined satellite retrievals of $R_{r s}$ values for use as inputs to retrieval algorithms. Again, both sets of retrievals were compared against the in situ sample measurements.

Results for NN retrievals are shown in Fig. 6 below. The left-hand side shows retrievals for all 41 measurements using in situ radiometric measurements as inputs compared against sample measurements. The top left-hand corner image shows retrievals using the $\mathrm{NN}$ algorithm with $443 \mathrm{~nm}$ as input. The bottom left-hand corner image shows these same retrievals using the $\mathrm{NN}$ algorithm, but now with $486 \mathrm{~nm}$ as input instead of $443 \mathrm{~nm}$. As can be seen, slightly better accuracies are obtained with the $486 \mathrm{~nm}$ input. The modest improvement in retrieval accuracies with $486 \mathrm{~nm}$ inputs is conjectured by us to be possibly due to lesser spectral interference from absorption at $486 \mathrm{~nm}$ than at $443 \mathrm{~nm}$ in some of the CDOM rich complex coastal waters included in the comparison.

On the top right-hand side, Fig. 6 shows the corresponding satellite NN retrievals for the same 41 measurements using satellite retrieved $443 \mathrm{~nm}$ as input. This shows a much lower retrieval accuracy than the left-hand image, which obtained by using in situ $R_{r s}$ values at $443 \mathrm{~nm}$. The results strongly suggest inaccurate satellite retrieved values of $R_{r s} 443$, possibly because of atmospheric correction inadequacies. This is further confirmed by a comparison of the satellite top right-hand corner as using $443 \mathrm{~nm}$ as input with the bottom right-hand corner, which shows satellite $\mathrm{NN}$ retrievals using $486 \mathrm{~nm}$ as input instead of $443 \mathrm{~nm}$. As can be seen, the $\mathrm{NN}$ retrievals with inputs of $486 \mathrm{~nm}$ instead of $443 \mathrm{~nm}$ achieve considerably higher retrieval accuracies, again probably due to larger atmospheric correction inadequacies contributing to

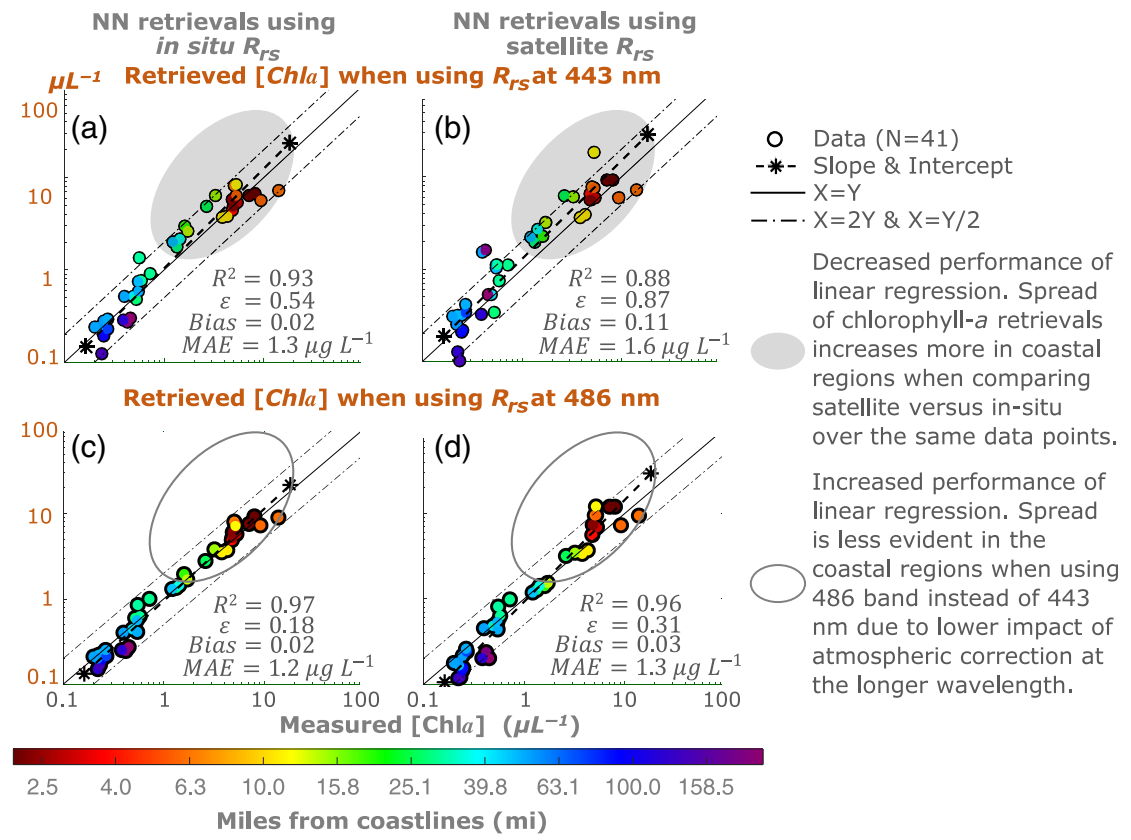

Fig. 6 Impact of using a deeper blue band at $443 \mathrm{~nm}$ instead of $486 \mathrm{~nm}$, as input to the NN retrieval algorithm for satellite and in situ radiometric retrieval accuracies of chlorophyll-a. Color bar relates retrieved [Chla] statistics to varying distance from the shoreline. 


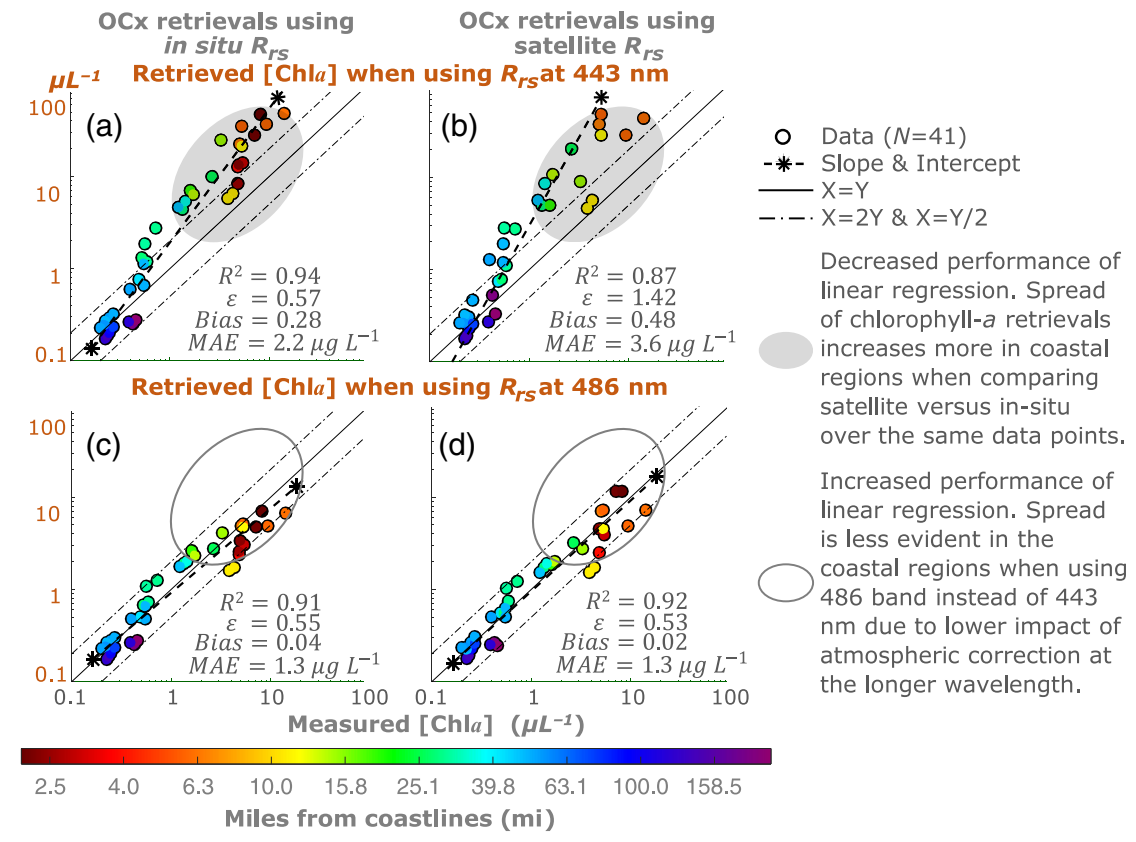

Fig. 7 Impact of using a deeper blue band at $443 \mathrm{~nm}$ instead of $486 \mathrm{~nm}$, as input to the OCl/OCx retrieval algorithm on satellite and in situ radiometric retrieval accuracies of chlorophyll-a. Color bar relates retrieved [Chla] statistics to varying distance from the shoreline.

larger errors for the $443 \mathrm{~nm}$ retrieval than for the $486 \mathrm{~nm}$ retrieval. It should be noted that this error would be in addition to the errors caused by possible spectral interference from CDOM absorption, affecting the in situ radiometric retrievals shown on the left-hand side of Fig. 6.

Similar results are obtained with retrievals using 443- and 486-nm bands as inputs to the OCI/OCx algorithms. These are shown in Fig. 7 below. The regression performances of both $\mathrm{NN}$ and $\mathrm{OCI} / \mathrm{OCx}$ algorithms are also summarized in Fig. 8 for comparison. All the percentage

\begin{tabular}{|c|c|c|c|c|c|c|c|c|c|}
\hline \multirow{3}{*}{$\begin{array}{l}\text { Bands used } \\
\quad(\mathrm{nm})\end{array}$} & \multirow{3}{*}{$\begin{array}{c}\mathrm{R}_{\mathrm{rs}} \text { input } \\
\text { source }\end{array}$} & \multicolumn{8}{|c|}{ Blue bands impact on the chlorophyll- $a$ retrieval accuracy $(\mathrm{N}=41)$} \\
\hline & & \multicolumn{4}{|c|}{ NN algorithm } & \multicolumn{4}{|c|}{ OCI/OCx algorithms } \\
\hline & & $\mathrm{R}^{2}$ & $\varepsilon$ & MAE & Bias & $\mathrm{R}^{2}$ & $\varepsilon$ & MAE & Bias \\
\hline \multirow{3}{*}{$\begin{array}{c}443 \\
551 \& 671\end{array}$} & in situ & 0.93 & 0.54 & 1.3 & 0.02 & 0.94 & 0.57 & 2.2 & 0.28 \\
\hline & satellite & 0.88 & 0.88 & 1.6 & 0.11 & 0.87 & 1.4 & 3.6 & 0.48 \\
\hline & PD & $5 \%$ & $24 \%$ & $8 \%$ & & $7 \%$ & $60 \%$ & $38 \%$ & \\
\hline \multirow{4}{*}{$\begin{array}{c}486 \\
551 \& 671\end{array}$} & in situ & 0.97 & 0.18 & 1.2 & 0.02 & 0.91 & 0.55 & 1.3 & 0.04 \\
\hline & satellite & 0.96 & 0.31 & 1.3 & 0.03 & 0.92 & 0.53 & 1.3 & 0.02 \\
\hline & PD & $1 \%$ & $9 \%$ & $2 \%$ & & $1 \%$ & $1 \%$ & $0 \%$ & \\
\hline & & \multicolumn{8}{|c|}{ Regression performance differences (486 - 443) } \\
\hline \multirow{2}{*}{486 vs. 443} & in situ & $+4 \%$ & $-25 \%$ & $-2 \%$ & & $-3 \%$ & $-1 \%$ & $-25 \%$ & \\
\hline & satellite & $+8 \%$ & $-41 \%$ & $-8 \%$ & & $+5 \%$ & $-62 \%$ & $-63 \%$ & \\
\hline \multirow{5}{*}{\multicolumn{3}{|c|}{$\begin{array}{l}\text { Parameters: } \\
\mathrm{N} \text { : Number of data points } \\
\mathrm{R}^{2} \text { : Coef. Of determination } \\
\varepsilon \text { : Error, sum of orthogonal distances. } \\
\text { MAE Mean Absolute Error } \\
\text { PD: Percentage Difference. }\end{array}$}} & \multicolumn{3}{|l|}{ Colo } & & & & \\
\hline & & & \multirow{2}{*}{$\begin{array}{l}\text { Reduced regression performance between in situ and } \\
\text { satellite chlorophyll-a retrievals when using } \mathrm{R}_{\mathrm{rs}} 443 \text {. }\end{array}$} & \multirow{3}{*}{\multicolumn{6}{|c|}{$\begin{array}{l}\text { Reduced regression performance between in situ and } \\
\text { satellite chlorophyll-a retrievals when using } \mathrm{R}_{\mathrm{rs}} 443 \text {. } \\
\text { Increased regression performance between in situ and } \\
\text { satellite chlorophyll-a retrievals when using } \mathrm{R}_{\mathrm{rs}} 486 \text {. }\end{array}$}} \\
\hline & & & & & & & & & \\
\hline & & & & & & & & & \\
\hline & & & & \multicolumn{6}{|c|}{$\begin{array}{l}\text { Percentage improve of chlorophyll-a retrievals for both } \\
\text { - in situ and satellite when using } R \quad 486 \text { instead of } 443\end{array}$} \\
\hline
\end{tabular}

Fig. 8 The impact of using a deeper blue band at $443 \mathrm{~nm}$ instead of $486 \mathrm{~nm}$, as input to the NN and $\mathrm{OCl} / \mathrm{OCx}$ retrieval algorithms on satellite and in situ radiometric retrieval accuracies of chlorophyll-a. 
differences are calculated relative to the highest value in each statistical parameter for both algorithms. Results shows that the exclusion of $443 \mathrm{~nm}$ in the algorithms inputs reduced the absolute errors by $8 \%$ to $63 \%$ and $2 \%$ to $25 \%$ for satellite and in situ radiometric retrievals, respectively. Additionally, the relative errors between satellite and in situ radiometric retrievals significantly decreased from $8 \%$ to $38 \%$ to values of $0 \%$ to $2 \%$ when using $486-\mathrm{nm}$ inputs instead of $443 \mathrm{~nm}$. Again, this tends to confirm that the use of longer wavelengths as inputs to the retrieval algorithms is advantageous, whether due to lesser vulnerability to inadequate atmospheric correction, and/or to lesser spectral interference from CDOM, and nonalgal particles, particularly in optically complex waters. These results are summarized in Fig. 8 below.

\subsection{Quantitative Examination of Temporal, Sampling Depth and Intrapixel Variabilities in KB HAB Waters of the WFS and their Impact on Retrieval Accuracies}

Our previous results, summarized in Sec. 2.2 above and reported in Refs. 1 and 2, implied that the accuracy and correlation of match-ups of VIIRS $K B$ HABs retrievals against near simultaneous sample measurements in the WFS were very vulnerable to relatively short-term temporal changes in the HABs scene being observed. We report here on a quantitative evaluation and impacts of these temporal variations, as well of variations with sampling depths and inter/ intra pixel variations carried out by means of detailed in situ sample field measurements in the WFS in conjunction with Mote Marine Laboratories in 2017 and 2018. Measurements were made along several transects, typically $2 \mathrm{~km}$ in length, off Sarasota, Florida. Many of the measurements were made at stations subpixel distances apart (generally $300 \mathrm{~m}$ ) on an outward leg and were then repeated for the same stations as closely as possible on a return leg. Radiometric and sample measurements were made at different depths: $0.1,0.5$, and $1 \mathrm{~m}$ for each station. Chlorophyll concentrations were obtained by HPLC analyses and $K B$ cell counts obtained by microscope analyses at Mote Marine Laboratories.

Figure 9(a) shows measured variabilities of $K B$ cell counts for eight stations (purple triangles) along a round trip transect over a 2-h period. The time between measurements at each numbered station is shown in minutes with the height of the triangle along the $Y$-axis being the magnitude of the coefficient of variation for that point. For station 7, with measurements 60 min apart, there is almost no variation, with a coefficient of variation close to zero, with the [Chla] holding steady (in the zoomed image) at $11 \mu \mathrm{g} \mathrm{L}^{-1}$ for the $60 \mathrm{~min}$, whereas for station 8 , there were three measurements, the second measurement being $60 \mathrm{~min}$ after the first, and the third measurement being another $60 \mathrm{~min}$ after the second, with corresponding changes in measured [Chla] (see zoomed image) and in coefficient of variation. As can be seen, temporal variations in $K B$ HABS concentrations are very common in the WFS with coefficients of variations of up to $22 \%$ being typical over periods of 20 to $60 \mathrm{~min}$.

Figure 9(b) shows the coefficient of variation of measured [Chla] across four VIIRS pixel sizes in the WFS. Three of the pixels had coefficients of variation of the order of $16 \%$ to $21 \%$. Pixel 3 was the highest, with a coefficient of $\sim 21 \%$, corresponding to a variation of [Chl $a]$ from $6.5^{1}$ to $10 \mu \mathrm{g} \mathrm{L}{ }^{-1}$ over a $230 \mathrm{~m}$ distance within the pixel (zoomed image). Pixel 4 had the lowest coefficient of variation of nearly zero, with a [Chla] of $2.8 \mu \mathrm{g} \mathrm{L}^{-1}$ remaining constant over a distance of $433 \mathrm{~m}$ in the pixel (zoomed image).

Figure 9(c) shows the variation with depth of measured [Chla] for transect of 11 stations. The black triangles show the coefficient of variation for each numbered station as the sampling depth is changed from 0.1 to $0.5 \mathrm{~m}$, with coefficients of variation typically in the range of $5 \%$ to $10 \%$. When the sampling depth range is increased from 0.5 to $1 \mathrm{~m}$, the coefficients of variation typically increase between $10 \%$ and $30 \%$. This suggests that a constant sampling depth of $0.25 \mathrm{~m}$ would be a reasonable practical goal for these types of waters.

These results highlight the temporal, intrapixel, and sampling depth variations that can typically occur in connection with retrievals in $K B$ HAB waters in the WFS. These can clearly have important impacts on attainable satellite retrieval accuracies, which merit further study. 

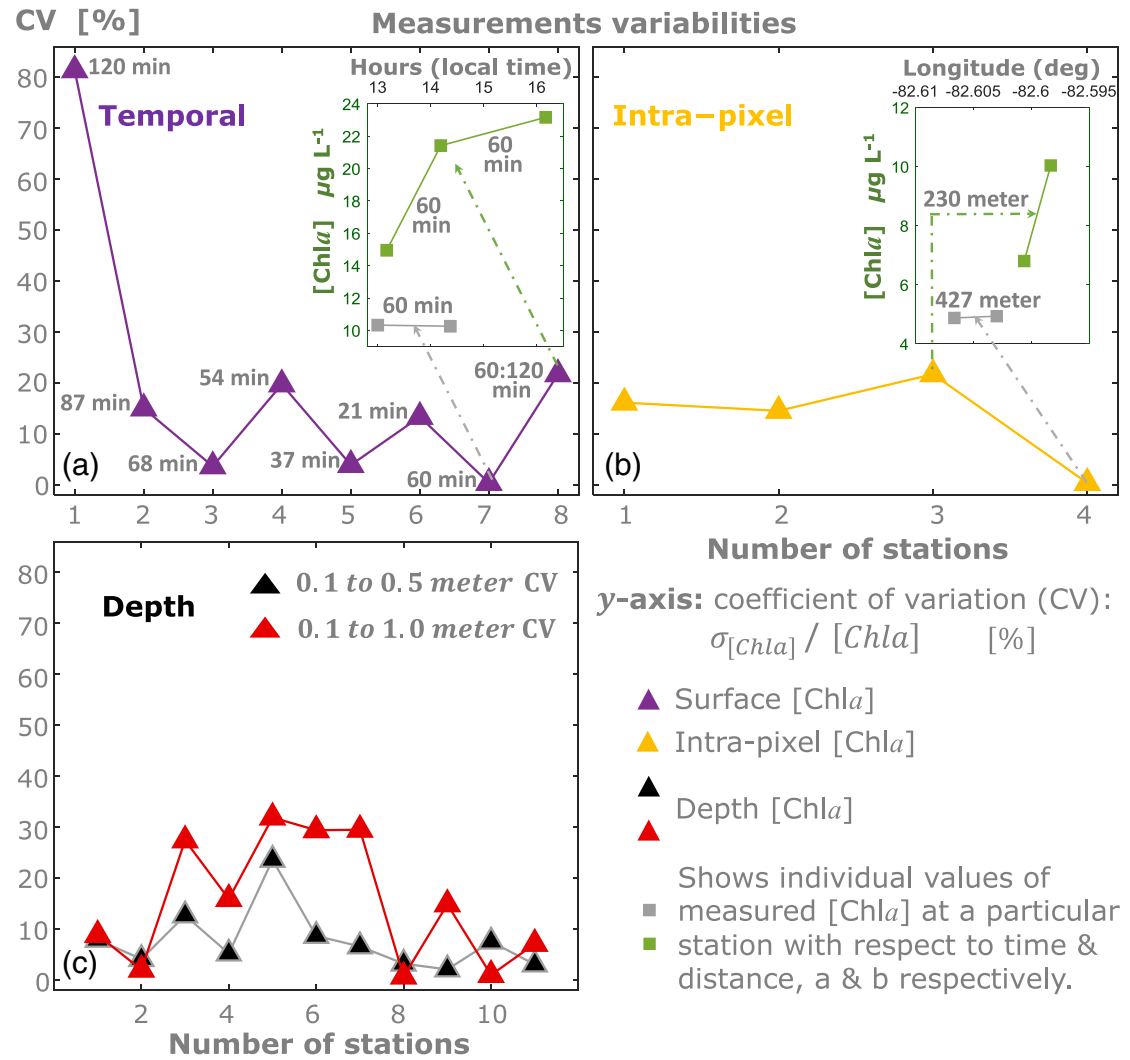

Number of stations

$y$-axis: coefficient of variation (CV):

$\sigma_{[\text {Chla }]} /[$ Chla $] \quad[\%]$

A Surface $[\mathrm{Chla}]$

$\Delta$ Intra-pixel [Chla]

$\Delta$

Depth $[$ Chla $]$

Shows individual values of

- measured $[\mathrm{Chl} a]$ at a particular

- station with respect to time \& distance, a \& b respectively.

Fig. 9 (a)-(c) Chlorophyll-a measurements variabilities in coastal bloom waters (chlorophyll-a values shown here are values equivalent to in situ measured $K B$ blooms cell counts). Results show variations ranged from $1 \%$ to $30 \%$ in average, due to temporal, inter-intra pixel and depth changes.

\subsection{Satellite Image Retrievals, and Observations of Variabilities in WFS}

Temporal variabilities in $K B$ HAB distributions in the WFS were also evident in consecutive satellite images available from VIIRS and MODIS satellites. For this purpose, changes in three consecutive overlapping satellite images of the WFS obtained on November 3, 2014, were studied. The first set of these images, Fig. 10, shows [Chla] concentrations of $K B$ HABs in Tampa Bay, Florida, as retrieved by the NN algorithm. Two of these images are from VIIRS, from first and second overpass, $96 \mathrm{~min}$ apart, plus an intermediate image is from MODIS-A, $70 \mathrm{~min}$ after the first VIIRS overpass image. The three images are overlaid with coincident $K B$ cell count measurements, which varied from nonexistent and low to high concentrations. We can focus on $\mathrm{NN}$ retrievals of [Chl $a$ ] from a small rectangular area in these three consecutive images, Figs. 10(a)-10(c). The three zoomed images at the top of the figure show the retrieved [Chla] distribution in the rectangle obtained from the consecutive VIIRS-MODIS-VIIRS images. The zoomed images show that concentrations are generally seen to be increasing, from zoomed image 10(a) to 10(b), then decreases again to 10(c), all within time intervals consistent with the temporal variations in situ field observations described above in Sec. 4.3 above. The changes in bloom concentrations observed may be due to drift, up- or downwelling, bloom growth or demise, and/or bloom swimming in vertical migrations. For reference, the lefthand side of Fig. 10 shows three zoomed open ocean, bloom-free areas, which as expected, remain unchanged for the three images. It should also be noted that Fig. 10(b) shows relatively high bloom concentration, even though an overlapping in situ measurement (denoted by "o" in the zoomed area) shows a very low or no bloom value. This may be explained by the occurrence of intrapixel variation, where a localized low value is overwhelmed by the averaging process apparently applicable to the retrieval of the whole pixel. An alternative explanation is an actual temporal variation in concentration that occurs between the time of the in situ measurement and 


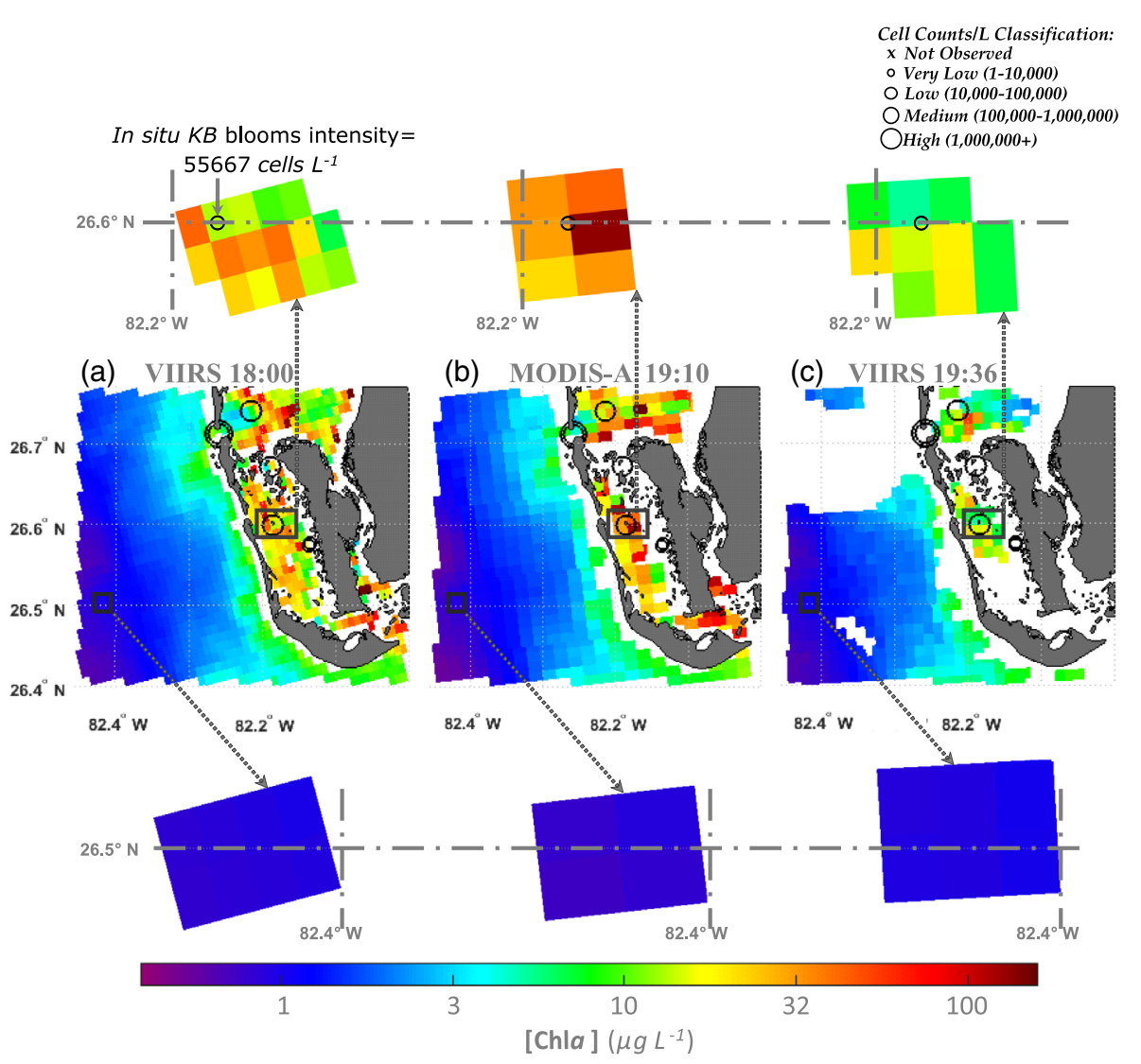

Fig. 10 Changes in $K B$ bloom for consecutive satellite images. Zoomed images (top row) show low intensity blooms of 55667 cell L $^{-1}$ (indicated by "o" symbol on the top row of the image) from in situ measurements and their retrievable surrounding pixels from VIIRS-MODIS-VIIRS overpasses. Zoomed images (bottom row) show nonbloom stable pixels nearby. (a) VIIRS 18:00, (b) MODIS-A 19:10, and (c) VIIRS 19:36.

the actual satellite observation. These results highlight the limitations on satellite retrievals potentially arising from both temporal and intrapixel variations.

A second set of three consecutive images is shown in Fig. 11 of another part of the Tampa Bay area. In these consecutive images, a bloom seems to shrink in [Chla] densities opposite to the wind and current direction, shown on the right of the figure, implying that there is a complexity of factors at work, possibly including downwelling and/or bloom swimming behavior, though there is no specific or quantitative evidence of linkage.

It is recognized that while consecutive satellite images can help qualitatively illustrate temporal changes and give an idea of their extent and concentration, it would be difficult to obtain quantitative useful information from them. It should also be noted that while consecutive overlapping images appear to show temporal changes, there is insufficient evidence from them to attribute the relative contributions of drift, patchiness, upwelling/downwelling or bloom swimming during vertical migration, or a combination of any of these causes to the changes observed. The quantitative field measurements results from Sec. 4.3 present much more solid results, confirming and quantifying $K B$ HABs inter-intra, temporal and depth variabilities as well as patchiness.

Finally, it should be noted that because of the low resolution of VIIRS and MODIS A satellites, with pixel sizes of 0.75 and $1.0 \mathrm{~km}$, respectively, retrievals from them are likely to miss many of the fine features associated with blooms. This can be seen in Fig. 12, where we have a much higher resolution RGB Landsat image of observation of blooms in Tampa Bay on March 18,2018 . These show a general view of the area, with a section shown zoomed on the upper right-hand side. This zoomed section can be contrasted with an image of the same zoomed area retrieved by NN from simultaneous VIIRS observations. With the much lower resolution 


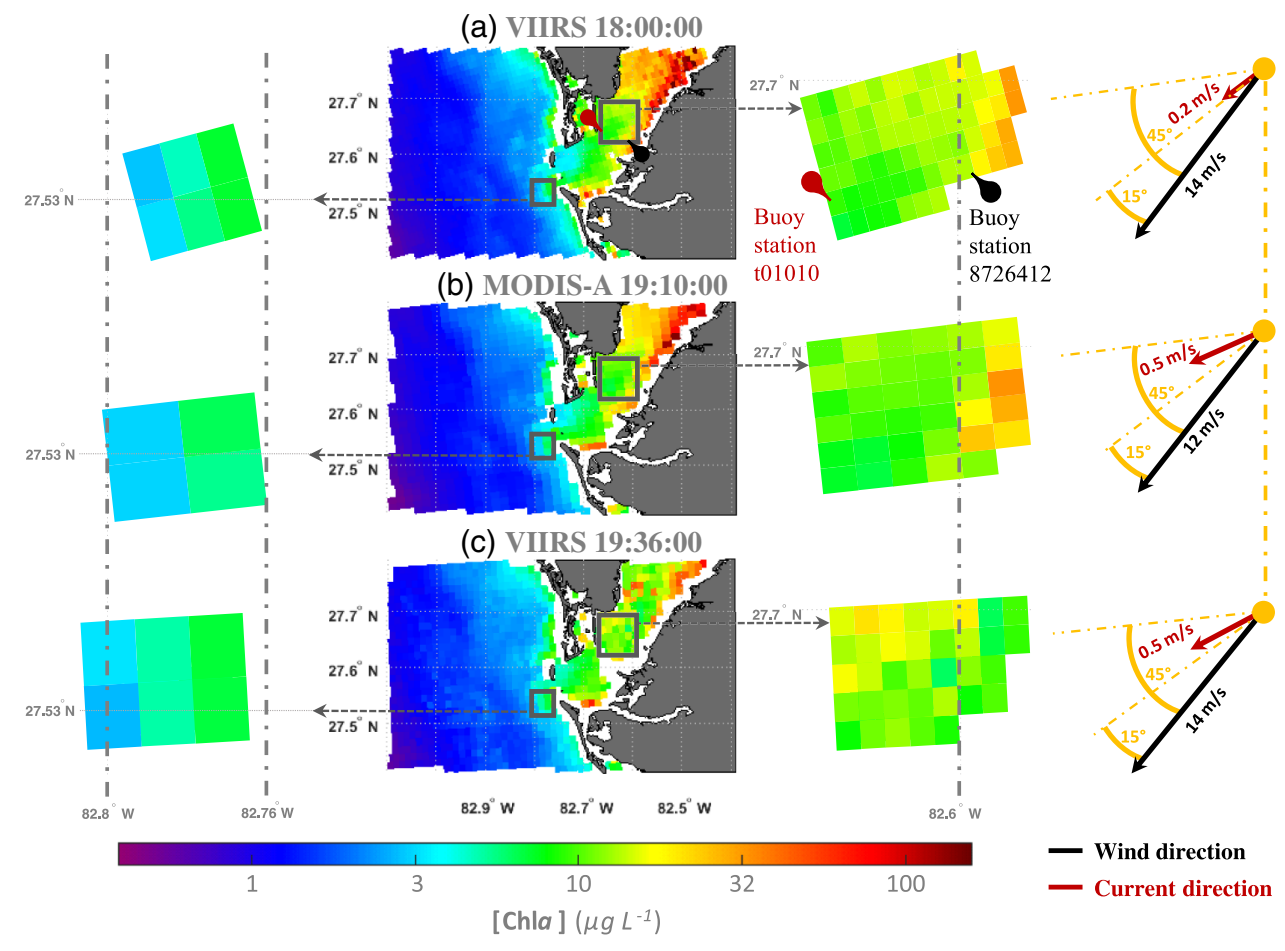

Fig. $11 \mathrm{NN}$ retrievals from Tampa Bay shows changes in $K B$ bloom for consecutive satellite images. (a) VIIRS 18:00:00, (b) MODIS-A 19:10:00, and (c) VIIRS 19:36:00.
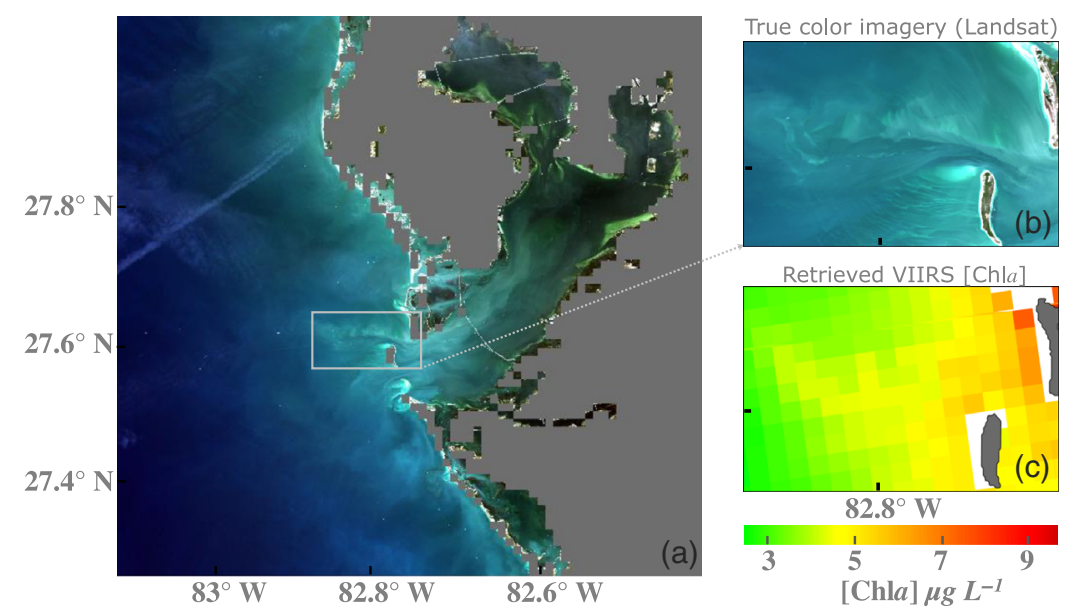

Fig. 12 Landsat RGB imagery during $K B$ bloom event on 03/18/2018, in WFS. (b) and (c) $K B$ bloom and ocean features that are inadequately observed through VIIRS [Chla] imagery.

available from VIIRS retrievals, it is seen that many of the fine details available in the Landsat image are missed. This suggests possibilities for exploring the combination of VIIRS retrievals with images from Landsat or drones to obtain additional detailed information.

\section{Discussion}

To answer questions regarding general applicability and potential advantages of our NN approach in waters beyond the WFS, in this paper, we extended NN retrieval comparisons to locations well beyond the WFS, to include both complex coastal and open ocean waters along the Florida and Atlantic coasts, which encompass different water types with a large dynamic range of chlorophyll- $a$ values. 
The NN retrievals were compared to retrievals using (OCIOCx), ${ }^{20,21}$ QAA, ${ }^{16,25,26}$ and RGCI. ${ }^{27}$ Measurements and retrievals were carried out in NOAA dedicated VIIRS OC $\mathrm{Cal} /$ Val cruises and our own cruises with Mote Marine Laboratory in the WFS over the 2014 to 2018 period. Most importantly, in these retrieval comparisons, in addition to extending locations and water types studied, we now also added in situ $R_{r s}$ radiometric measurements, to complement the in situ sampling. Since these $R_{r s}$ measurements are in situ, they are not subject to the impact of possibly inadequate (or incorrect) correction procedures that are applied to atmospheric transmission assumptions and that can erroneously impact satellite $R_{r s}$ retrievals and hence retrieval accuracies. Instead, by using these in situ $R_{r s}$ measurements as inputs to the different retrieval algorithms, the retrieval comparisons obtained in this manner are probably a better indication of the relative intrinsic retrieval accuracies and merits of the algorithms being compared.

In addition to the above retrieval comparisons from in situ radiometric measurements, comparisons were made of simultaneous satellite retrievals against simultaneous colocated sample measurements using the different algorithms. As well as permitting us to rank the satellite retrieval accuracies and efficacies of NN and other algorithms for waters beyond the WFS, this permits us to examine and isolate the impacts of different factors on satellite retrieval accuracies.

We next investigated the impact of using deep blue bands to obtain $R_{r s}$ on satellite retrieval accuracies. Since it is conjectured that in satellite retrievals, it is the deep blue wavelengths that are more significantly affected by atmospheric correction inadequacies, we next zeroed in on the impacts on algorithm retrieval accuracy when deep blue wavelengths are used for retrieving $R_{r s}$ values. For this work, we compared algorithm retrievals from by far the two best performing algorithms, $\mathrm{NN}$ and $\mathrm{OCI} / \mathrm{OCx}$ against in situ sample measurements, using both in situ radiometric measurements as inputs, and satellite retrievals, with each of the algorithms using for blue wavelengths either $443 \mathrm{~nm}$ (deep blue) or $486 \mathrm{~nm}$ (nondeep blue). These combined comparisons allow us to isolate the impact of atmospheric correction inadequacies, as well as identify possible underwater spectral interference in CDOM rich and complex waters.

Other factors that were quantitatively studied included short-term temporal, intrapixel, and sample depth variabilities, and their impact on retrieval accuracies was examined and confirmed, in field studies, as well as from images obtained from consecutive satellite overpasses.

\section{Conclusions}

The extension of NN retrieval comparisons to examine their applicability to locations well beyond the WFS, and including both complex coastal and open ocean waters along the Florida and Atlantic coasts, which encompass different water types with a large dynamic range of chlorophyll- $a$ values compared, yielded the following results:

i. The comparison of retrievals using in situ $R_{r s}$ radiometric measurements as inputs to retrieval algorithms validated against in-situ sample measurements showed that NN retrievals again evidenced the highest retrieval accuracy statistics for widely varying waters beyond the WFS region. NN retrieval accuracies were closely followed by those for $\mathrm{OCI} / \mathrm{OCx}$, then more distantly by QAA and RGCI, particularly in optically complex and shallow waters. Since the use of in-situ radiometric measurements eliminates the potential impact of inadequate atmospheric corrections, this result is more indicate of the intrinsic retrieval accuracy of the algorithms.

ii. Similar results were achieved when comparing the available VIIRS satellite retrievals over the same different waters types. Both results unambiguously confirmed $\mathrm{NN}$ efficacy and viability in widely varying waters beyond the WFS and were a testament to the effective training of $\mathrm{NN}$ on the 10,000 unfettered and randomly simulated water constituents within the widest permissible range of values. The results also argue for a more general investigation of the suitability of adoption of $\mathrm{NN}$ retrieval algorithms on a global scale.

The results of the comparisons on the use of deep blue bands in both NN and OCI/OCx retrieval algorithms clearly showed that for satellite retrievals validated against in situ sample measurements, both algorithms gave better retrieval accuracies when the longer 486-nm band 
was used instead of the deeper blue 443-nm band. The inference being that the improvement is due to inadequate atmospheric transmission corrections (i.e., bigger errors) and/or instrumental limitations at $443 \mathrm{~nm}$ compared with $486 \mathrm{~nm}$. Improvements were also seen for the longer wavelengths when in situ radiometric values were used as inputs to the retrieval algorithms. In this case, this is attributed to likely higher underwater spectral interference at $443 \mathrm{~nm}$ than $486 \mathrm{~nm}$ in CDOM rich and complex coastal waters. The comparisons on the use of deep blue bands are also repeated for the OCI/OCX algorithms, which has options for either $443 \mathrm{~nm}$ or 486 inputs, and similar results were achieved. The results argue for the avoidance of deep blue bands in satellite OC retrieval algorithms and the use of longer wavelengths.

The fact that for the optically complex and shallow waters the NN algorithm exhibited higher retrieval accuracy statistics would tend to support the notion that this may be at least partially due to the use of longer $R_{r s}$ input wavelengths for $\mathrm{NN}$, than the deeper blue wavelengths used in other algorithms, thereby possibly lessening the impact on measured $R_{r s}$ values of spectral interference by dissolved organic matter (CDOM), and nonalgal particles (both expected in more complex waters). It should also be noted that the NN output of $\left(a_{p h}\right)$ is at the 443-nm band, which is at the peak of phytoplankton absorption, and thus exhibits the most sensitive variation in natural water. Furthermore, the NN approach does not require measurements at the shorter wavelength channels, which would be typically most impacted by atmospheric correction difficulties if actual satellite measurements in the deep blue (443 nm) band were required. These factors can be expected to contribute significantly to the sensitivity and accuracy of the NN retrievals of $a_{p h}$ (443) parameter, particularly in optically complex waters, In any case, it can be unambiguously concluded from results presented here, that the intrinsic efficacy of NN retrieval algorithms extends well beyond the confines of the WFS region to widely varying waters types, and argues for its adoption as a product for such retrievals.

Quantitative studies of other factors including short-term temporal, intrapixel, and sample depth variabilities, and their impact on retrieval accuracies were examined and confirmed, in field studies, as well as from images obtained from consecutive satellite overpasses. These results highlight the limitations, which these factors place on the accuracy and utility of satellite OC retrievals, particularly in complex coastal waters and suggest the complementary use of other higher resolution observation platforms, possibly including unmanned aerial vehicles.

\section{Appendix}

\subsection{NN Background}

For the development of the NN algorithm, ${ }^{1,2,4-8}$ a synthetic dataset of 20,000 IOPs was randomly simulated within the very wide range of parameters exhibited in the NASA bio-optical marine algorithm dataset. ${ }^{12}$ These simulated IOPs, whose range and variability is well represented in the literature, ${ }^{13-17}$ were then used as inputs to a four-component bio-optical model, ${ }^{7,16,17}$ which in conjunction with a HydroLight based, ${ }^{18}$ parameterized forward model, described in Lee et al., ${ }^{16}$ and produced 20,000 sets of $R_{r s}$ values at 486, 551, and $671 \mathrm{~nm}$ (for VIIRS) and at 488, 555, and $667 \mathrm{~nm}$ for MODIS. The NN was trained on 10,000 of these values and tested on the 10,000 remaining subset, as well as on field data to solve the inverse problem ${ }^{19}$ of retrieving OC physical parameters, including $a_{\mathrm{ph} 443}$, from $R_{r s}$ values. The NN algorithm uses $R_{r s}$ input at 486, 551, and $671 \mathrm{~nm}$, values used at a longer $\lambda$, which are not greatly impacted by atmospheric correction. NN produces output of phytoplankton absorption $\left(a_{p h}\right)$ at 443 . Output is at the peak of $a_{p h}$ thus exhibits most variation (Fig. 13).

\subsection{NN Implementation}

NN implementation to retrieve $a_{p h} 443$ is shown in Fig. 14 below. The first step requires inputs of remote sensing reflectance's $\left[R_{r s}(\lambda)\right]$ at 486,551 , and $671 \mathrm{~nm}$, all expressed as base 10 logarithms, and standardized by removing the mean $(\mu)$, then scaling the difference by the standard deviation $(\sigma)$ of the simulated dataset for each $R_{r s}\left(\lambda_{i}\right)$ input. In the final step, the output ( $a_{p h} 443$ ) is denormalized to obtain meaningful results, as shown in bottom of Fig. 14 (output). The NN is 


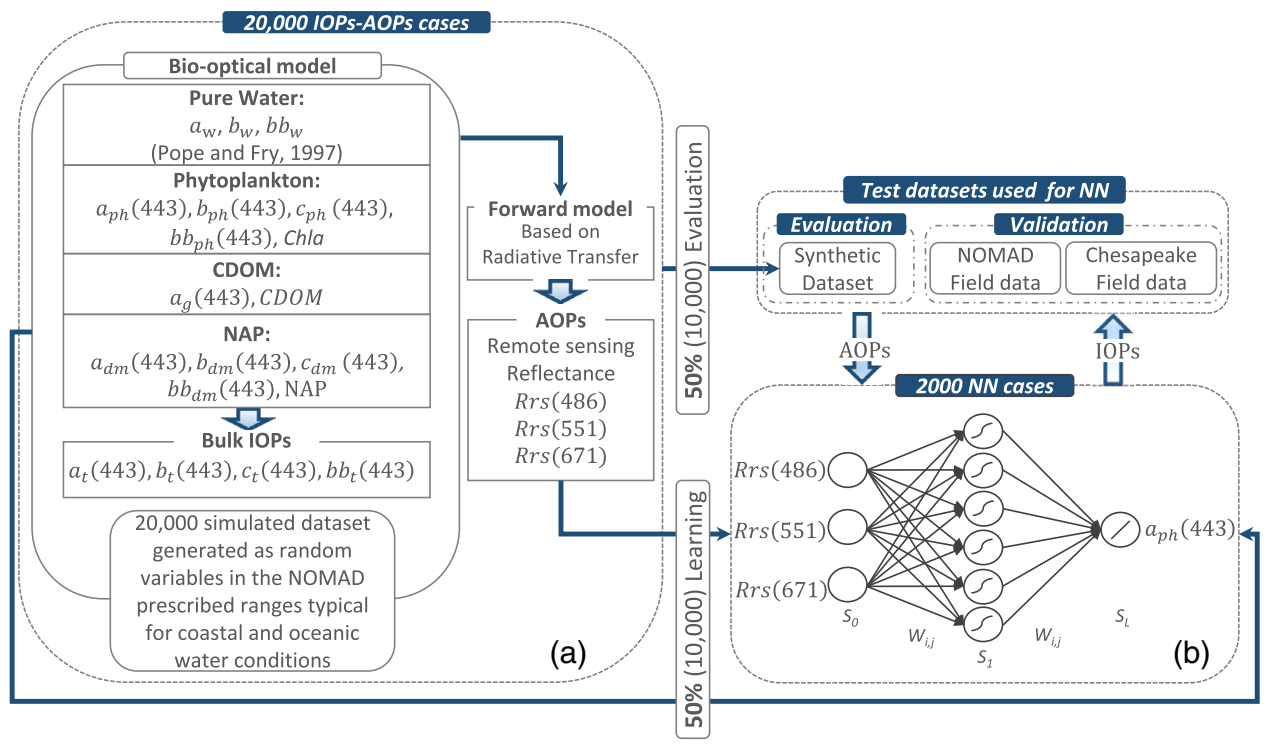

Fig. 13 Development of the NN algorithm. (a) The bio-optical model and forward model simulations and (b) the architecture of the $\mathrm{NN}$, one-hidden layer multilayer perceptron, trained with 10,000 set of $R_{r s}$ and related IOPs.

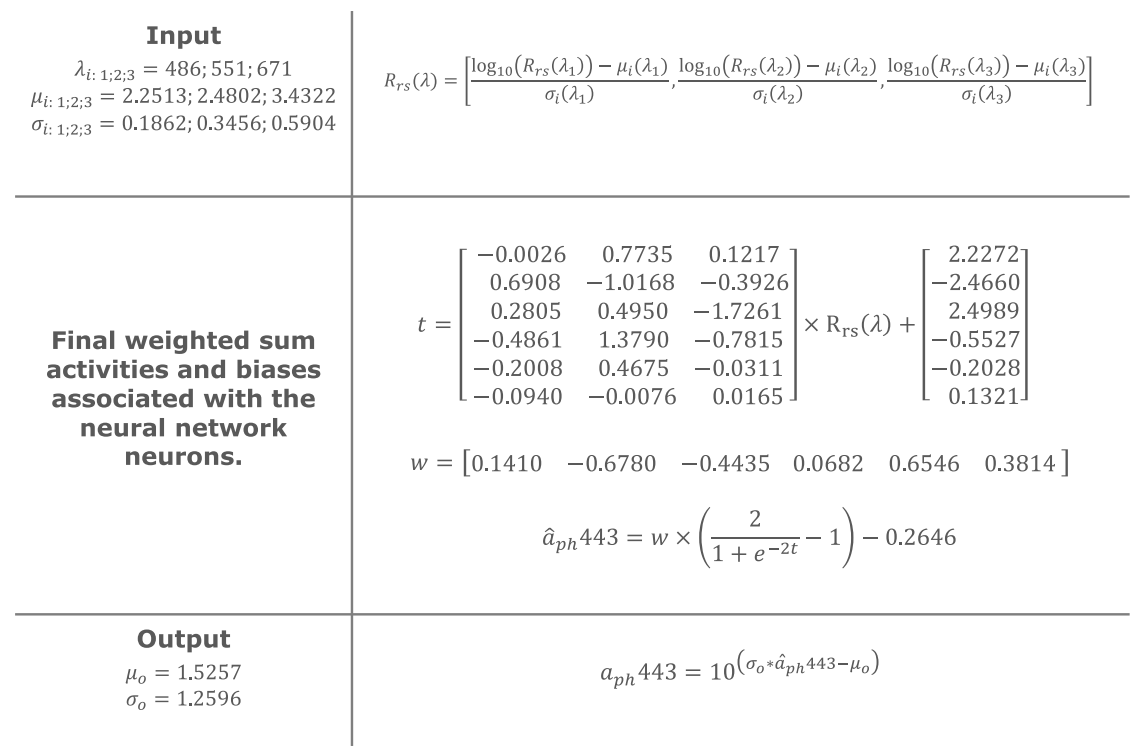

Fig. 14 The NN implementation for $a_{p h} 443\left(\mathrm{~m}^{-1}\right)$ retrievals.

trained using normalized $R_{r s}(\lambda)$ values so that it is equally sensitive to all inputs, avoiding conditioning problems.

The term $\left(\frac{2}{1+e^{-2 t}}-1\right)$ in Fig. 14 represents the NN hyperbolic sigmoid activation function and is used to transfer the final weighted sum activity and biases associated with each computational neuron in the trained NN. More details of the NN algorithm architecture can be found in Refs. 1, 2, 4, and 7.

The $a_{p h} 443$ can then be converted to [Chla], assuming a constant specific absorption value or using any value of specific phytoplankton absorption, which is area specific. ${ }^{9}$

\section{Acknowledgments}

This work was partially performed while Dr. Ahmed El-Habashi held an NRC Research Associateship award at the U.S. Naval Research Laboratory in Washington, DC, funded under 
contractor number N00173-17-2-C002. The work was also supported by grants from NOAA through NOAA CREST and the Joint Polar Satellite System (JPSS) for the NOAA OC calibration and validation $(\mathrm{Cal} / \mathrm{Val})$ project. Some of the in situ data used in this study were collected from the NOAA dedicated VIIRS OC Cal/Val cruises supported by the JPSS program, the NOAA Office of Marine and Aviation Operations. The chlorophyll- $a$ and sample data were collected and processed by the Ocean Biology Processing Group (OBPG) at NASA Goddard Space Flight Center, and by the phytoplankton ecology staff at the Mote Marine Laboratory.

\section{References}

1. A. El-Habashi et al., "Satellite retrievals of Karenia brevis harmful algal blooms in the west Florida shelf using neural networks and comparisons with other techniques," Remote Sens. 8(5), 377 (2016).

2. A. El-Habashi et al., "Satellite retrievals of Karenia brevis harmful algal blooms in the west Florida shelf using neural networks and impacts of temporal variabilities," J. Appl. Remote Sens. 11(3), 032408 (2017).

3. S. Ahmed et al., "Evaluation and comparison of JPSS VIIRS neural network retrievals of harmful algal blooms with other retrieval algorithms, validated against in-situ radiometric and sample measurements in the west Florida shelf, and examination of impacts of atmospheric corrections, temporal variations and complex in-shore waters," Proc. SPIE 10784, 1078402 (2018).

4. A. El-Habashi, "Remote sensing over coastal and open oceans: retrieval of water constituents from scalar and polarimetric observations," PhD Thesis, The City College of New York, (2018).

5. I. Ioannou et al., "Neural network approach to retrieve the inherent optical properties of the ocean from observations of MODIS," Appl. Opt. 50(19), 3168-3186 (2011).

6. I. Ioannou et al., "Deriving ocean color products using neural networks," Remote Sens. Environ. 134, 78-91 (2013).

7. I. Ioannou et al., "Remote estimation of in water constituents in coastal waters using neural networks," Proc. SPIE 9240, 92400T (2014).

8. A. El-Habashi and S. Ahmed, "Neural network algorithms for retrieval of harmful algal blooms in the west Florida shelf from VIIRS satellite observations and comparisons with other techniques, without the need for a fluorescence channel," Proc. SPIE 9638, 96380B (2015).

9. C. Le and C. Hu, "A hybrid approach to estimate chromophoric dissolved organic matter in turbid estuaries from satellite measurements: a case study for Tampa Bay," Opt. Express 21(16), 18849-18871 (2013).

10. J. P. Cannizzaro et al., "Detection of Karenia brevis blooms on the west Florida shelf using in situ backscattering and fluorescence data," Harmful Algae 8(6), 898-909 (2009).

11. I. M. Soto et al., "Evaluation and optimization of remote sensing techniques for detection of Karenia brevis blooms on the west Florida shelf," Remote Sens. Environ. 170, 239-254 (2015).

12. P. J. Werdell and S. W. Bailey, "An improved in-situ bio-optical data set for ocean color algorithm development and satellite data product validation," Remote Sens. Environ. 98(1), 122-140 (2005).

13. C. D. Mobley, Light and Water: Radiative Transfer in Natural Waters, Academic Press, San Diego (1994).

14. R. M. Pope and E. S. Fry, "Absorption spectrum $(380-700 \mathrm{~nm})$ of pure water. II. Integrating cavity measurements," Appl. Opt. 36(33), 8710-8723 (1997).

15. A. M. Ciotti, M. R. Lewis, and J. J. Cullen, "Assessment of the relationships between dominant cell size in natural phytoplankton communities and the spectral shape of the absorption coefficient," Limnol. Oceanogr. 47, 404-417 (2002).

16. Z. Lee, K. L. Carder, and R. A. Arnone, "Deriving inherent optical properties from water color: a multiband quasi-analytical algorithm for optically deep waters," Appl. Opt. 41(27), 5755-5772 (2002). 
17. Z. Lee, "Reports of the international ocean-colour coordinating group: fundamentals, tests of algorithms, and applications," Report 5 (2006).

18. C. D. Mobley and L. K. Sundman, "Hydrolight 4.2 technical documentation," Technical Report, Sequoia Scientific, Incorporated, Redmond, Washington (2001).

19. F. Aires et al., "A new neural network approach including first-guess for retrieval of atmospheric water vapor, cloud liquid water path, surface temperature and emissivities over land from satellite microwave observations," J. Geophys. Res. Atmos. 106(D14), 14,887-14,907 (2001).

20. C. Hu, Z. Lee, and B. Franz, "Chlorophyll-a algorithms for oligotrophic oceans: a novel approach based on three-band reflectance difference," J. Geophys. Res. Ocean 117(C1), 1978-2012 (2012).

21. J. E. O'Reilly et al., "Ocean color chlorophyll algorithms for SeaWiFS," J. Geophys. Res. Ocean 103(C11), 24937-24953 (1998).

22. NASA's OceanColor Web, "Chlorophyll-a algorithm," NASA's OceanColor Web by the Ocean Biology Processing Group (OBPG) at NASA's Goddard Space Flight Center, https://oceancolor.gsfc.nasa.gov/atbd/chlor_a/.

23. J. Werdell, "Global bio-optical algorithms for ocean color satellite applications: inherent optical properties algorithm workshop at ocean optics XIX; Barga, Italy, 3-4 October 2008," EOS Trans. Am. Geophys. Union 90(1), 4 (2009).

24. P. J. Werdell et al., "Generalized ocean color inversion model for retrieving marine inherent optical properties," Appl. Opt. 52(10), 2019-2037 (2013).

25. Z. Lee et al., "An update of the quasi-analytical algorithm (QAA_v5)," International Ocean Color Group Software Report, pp. 1-9 (2009).

26. Z. Lee and K. L. Carder, "Absorption spectrum of phytoplankton pigments derived from hyperspectral remote-sensing reflectance," Remote Sens. Environ. 89(3), 361-368 (2004).

27. L. Qi et al., "VIIRS observations of a Karenia brevis bloom in the Northeastern Gulf of Mexico in the absence of a fluorescence band," IEEE Geosci. Remote Sens. Lett. 12(11), 2213-2217 (2015).

28. R. Arnone et al., "Diurnal changes in ocean color in coastal waters," Proc. SPIE 9827, 982711 (2016).

29. M. Ondrusek and V. P. Lance, "Report for dedicated JPSS VIIRS ocean color calibration/ validation cruise," NOAA technical report NESDIS; 146 (2015).

30. M. Ondrusek et al., "Report for dedicated JPSS VIIRS ocean color December 2015 calibration/validation cruise," NOAA Technical Report NESDIS; 148 (2016).

31. M. Ondrusek et al., "Report for dedicated JPSS VIIRS ocean color calibration/validation cruise October 2016," NOAA Technical Report NESDIS (2017).

32. L. Leng et al., "Ordinary least square regression, orthogonal regression, geometric mean regression and their applications in aerosol science," J. Phys. Conf. Ser. 78, 012084 (2007).

33. P. J. Franks, "Spatial patterns in dense algal blooms," Limnol. Oceanogr. 42(5), 1297-1305 (1997).

34. R. Stumpf et al., "Monitoring Karenia brevis blooms in the Gulf of Mexico using satellite ocean color imagery and other data," Harmful Algae 2(2), 147-160 (2003).

Ahmed El-Habashi received his MS and $\mathrm{PhD}$ degrees in electrical engineering from the City University of New York. His thesis explored ocean color algorithm development using machine learning techniques, as well as the light-polarization properties of oceanic coastal waters and their relationship to fluorescence. He currently holds an NRC Research Associateship award at the U.S. Naval Research Laboratory in Washington, DC. His research area focuses on retrievals of seawater optical properties using measured polarized light filed and vector radiative transfer codes.

Sam Ahmed is Herbert Kayser professor of electrical engineering and director of the NOAA CREST Optical Remote Sensing Laboratory at City College, New York. He was educated at the Universities of Cambridge and London. During his long career in industry and academia, he has been active in laser development, LiDAR atmospheric remote sensing, and in satellite remote sensing of ocean color. More recently, he has focused on neural network algorithms for the satellite detection of harmful algal blooms in the WFS. 
Michael Ondrusek is an oceanographer for the NOAA Center for Satellite Applications and Research (STAR). He served as the Division Ocean Color Science Team Lead and the Ocean Color Product Oversight panel cochair from 2006 to 2011, the Marine Optical Buoy PI from 2007 to 2010, and currently is the JPSS VIIRS Cal/Val annual cruise chief scientist. His research interests include ocean color calibration and validation, satellite ocean color product algorithm development, and coastal ecology.

Vincent Lovko manages the Phytoplankton Ecology Research Program at Mote Marine Laboratory in Sarasota, Florida. He leads Mote's co-operative red tide program, which conducts red tide monitoring and research in collaboration with the state of Florida, providing data for red tide status updates as well as other collaborative data products. His program's research focuses on Karenia brevis detection technologies, mitigation strategies, and bloom dynamics in relation to water column variables and phytoplankton community structure. 